\title{
UCRL-JC-149205
}

LAWRENCE LIVERMORE NATIONAL LABORATORY

\section{Materials Science Under Extreme Conditions of Pressure and Strain Rate}

B. A. Remington, G. Bazan, E. Bringa, M.

Caturla, M. J. Edwards, S. G. Glendinning, B. Kad, D. H. Kalantar, M. Kumar, B. F. Lasinski, K. T. Lorenz, J. McNaney, D. Meyerhofer, M. A. Meyers, S. M. Pollaine, D. B. Reisman, D. Rowley, M. Schneider, J. Stoklen, J. Wark, B. Yaakobi

\section{March 27, 2003}

Special Symposium in Honor of Ronald W. Armstrong, The $132^{\text {nd }}$ Annual Meeting of TMS, San Diego, CA March 2-6, 2003 
This document was prepared as an account of work sponsored by an agency of the United States Government. Neither the United States Government nor the University of California nor any of their employees, makes any warranty, express or implied, or assumes any legal liability or responsibility for the accuracy, completeness, or usefulness of any information, apparatus, product, or process disclosed, or represents that its use would not infringe privately owned rights. Reference herein to any specific commercial product, process, or service by trade name, trademark, manufacturer, or otherwise, does not necessarily constitute or imply its endorsement, recommendation, or favoring by the United States Government or the University of California. The views and opinions of authors expressed herein do not necessarily state or reflect those of the United States Government or the University of California, and shall not be used for advertising or product endorsement purposes.

This work was performed under the auspices of the U.S. Department of Energy by University of California, Lawrence Livermore National Laboratory under Contract W-7405-Eng-48. 
Materials science under extreme conditions of pressure and strain rate*

B.A. Remington, ${ }^{1}$ G. Bazan, ${ }^{1}$ E. Bringa, ${ }^{1}$ M. Caturla, ${ }^{1}$ M.J. Edwards, ${ }^{1}$ S.G. Glendinning, ${ }^{1}$

B. Kad, ${ }^{2}$ D.H. Kalantar, ${ }^{1}$ M. Kumar, ${ }^{1}$ B.F. Lasinski, ${ }^{1}$ K.T. Lorenz, ${ }^{1}$ J. McNaney, ${ }^{1}$

D. Meyerhofer, M.A. Meyers, ${ }^{2}$ S.M. Pollaine, ${ }^{1}$ D.B. Reisman, ${ }^{1}$ D. Rowley, ${ }^{1}$ M.

Schneider ${ }^{2}$, J. Stolken, ${ }^{1}$ J. Wark ${ }^{3}$, B. Yaakobi ${ }^{4}$

${ }^{1}$ Lawrence Livermore National Laboratory, ${ }^{2}$ University of California - San Diego, ${ }^{3}$ Oxford University, ${ }^{4}$ Laboratory for Laser Energetics

(March 26, 2003, 3:20 pm)

\section{Abstract}

Solid state dynamics experiments at very high pressures $(\mathrm{P}>>10 \mathrm{GPa})$ and strain rates $(\dot{\varepsilon}$ $>10^{5} \mathrm{~s}^{-1}$ ) have been demonstrated on high energy laser facilities, albeit over brief intervals of time and small spatial scales. We have developed two methods for driving samples to high pressures $(10-100 \mathrm{GPa})$ at high strain rate $\left(10^{6}-10^{8} \mathrm{~s}^{-1}\right)$ in the solid state. One method uses a shockless compression technique, and the other uses multiple staged shocks. These drives are calibrated with VISAR measurements of the resulting compression wave. Deformation mechanisms are inferred under these conditions by characterizing recovered samples. Material strength at high pressures and strain rates is deduced by measuring the reduced growth of material perturbations at a hydrodynamically unstable interface. Microscopic lattice response is determined by time-resolved Bragg diffraction and x-ray absorption spectroscopy (EXAFS). Largescale simulations, both at the continuum level using constitutive models and at the lattice level using molecular dynamics simulation, are used to interpret these integral experiments. We will review our progress in this new area of laser-based materials science research, then present a vision for carrying these solid-state experiments to much higher pressures, $\mathrm{P}>1000 \mathrm{GPa}$, on the National Ignition Facility (NIF) laser facility.

\section{INTRODUCTION}

High strain rate materials dynamics and deformation mechanisms have been a topic of great interest for decades. [Kocks, Argon, \& Ashby, 1975; Frost \& Ashby, 1982; Meyers, 
1994; Murr; Hull \& Bacon, 1984; Hirth \& Lothe, 1982] Materials response to shocks and other high strain rate deformation has led to a number of theories, both empirical, and more recently physically based. In particular, there historically has been and continues to be great interest in developing and testing constitutive models that allow continuum hydrodynamic computer to simulate plastic flow in the solid state. Models such as the Johnson-Cook, [Johnson, 1983] Zerilli-Armstrong, [Zerilli, 1990 ] Mechanical Threshold Stress (MTS), [Follansbee, 1991; 1986] Steinberg-Lund, [Steinberg, 1989] and Steinberg-Guinan [Steinberg, 1980] models are widely used in the materials dynamics community. These models have typically been tested or experimentally calibrated in experiments on Hopkinson bars, Taylor cylinders, or with HE-driven shock or compression waves at pressures of up to a few $10 \mathrm{~s}$ of GPa and strain rates of $10^{3}-10^{5} \mathrm{~s}^{-1}$. We describe here our efforts to create materials science experiments at much higher pressures and strain rates, where we anticipate new regimes and mechanisms of solidstate dynamics. To reach these unique conditions of materials dynamics, we use large laser facilities to generate carefully tailored pressure waves, diagnosed with a variety of time-resolved diagnostics.

The hope is that at ultrahigh strain rates and pressures, fundamental questions of materials deformation dynamics can be addressed. Examples of such fundamental issues that may be addressed with experiments at ultrahigh strain rate are:

1. Is there a "relativistic" regime at the highest $\dot{\varepsilon}$ (ie, is there an absolute limit on $\mathrm{v}_{\text {disloc }}$ )?

2. Are there upper limits on $\rho_{\text {disloc }}, \mathrm{d} \rho_{\text {dislod }} / \mathrm{dt}$ as $\mathrm{d} \varepsilon / \mathrm{dt}$ increases to very high values?

3. Do initial conditions matter at ultrahigh shear stresses and compressions?

4. Does deformation universally transition to Schmid's law at extreme $\dot{\varepsilon}$

5. Is phonon drag the dominant deformation mechanism at ultrahigh strain rates?

6. How does the Peierls-Nabarro stress scale to ultrahigh pressures?

7. Does material strength scale with shear modulus to extreme pressures and $\dot{\varepsilon}$ ?

8. What is the time scale for solid-to-solid, solid-to-liq., liq.-to-solid phase transitions?

9. Is there a natural length scale in plastic flow at very high strain gradient? 
In thie paper we give an overview of our efforts to create an ultrahigh pressure-strain rate deformation dynamics testbed. Three companion papers [Schneider; McNaney; Bringa, these proceedings, 2003] go into more depth about key parts of this research. Our paper is organized as follows. We discuss constitutive models in Sec. II. In Sec. III we describe the new drive mechanisms, and in Sec. IV we describe deformation dynamics at these regimes. In Sec. $\mathrm{V}$ we discuss material strength at very high pressures and strain rates, and in Sec. VI present results on measuring lattice response. We discuss the outlook for the future in Sec. VII.

\section{Constitutive Models}

To facilitate the discussion that follows and to identify key aspects to the deformation dynajics, we begin by deriving a simple constitutive model. The fundamental assumption of the model is that dislocation transport is the microscopic mechanism underlying dynamic deformation. Resistance to dislocation transport is then what we macroscopically refer to as material strength. Assume that there exists a population of potentially mobile dislocations in a sample, but that the vast majority are pinned against obstacles. An applied shear stress is required to initiate deformation by pushing dislocations over or through their barriers, so that they can move. The stronger the barrier, the greater the required applied shear stress to achieve a given deformation, characterized by $(\varepsilon, \dot{\varepsilon})$, and hence the stronger the material. The applied stress required to initiative deformation is temperature dependent, since thermal fluctuations can assist in helping dislocations surmount, cut through, or bypass a barrier. This mode of deformation is referred to as stress assisted thermal activation, and is schematically illustrated in Fig. 1a. [Hull \& Bacon, 1984] An edge dislocation is depicted pinned against a barrier at a height corresponding to the applied shear stress. At zero temperature, the stress required to completely surmount the barrier (that is, with no thermal assist) is equal to the barrier height, which we denote the Peierls barrier or Peierls-Nabarro barrier, $\sigma_{0}$. At zero temperature, the work that most be expended to surmount or cut through the barrier is proportional to the area under the barrier stress curve, and corresponds to the activation energy, $\Delta \mathrm{F}$. 
Consider the dislocation pinned against the obstacle, as shown in Fig. 1a, and assume that the sample is at temperature $T$. The dislocation has a thermal vibration frequency, $v_{0}$, which can be considered as the frequency of attempts to jump the barrier. The frequency of successful jumps over the boundary, $v_{1}$, can be written by the so-called Arrhenius equation,

$$
v_{1}=v_{0} e^{-\Delta F / k T}
$$

where $\Delta \mathrm{F}$ is the activation energy. If the average distance between pinning obstacles is $\lambda$, and the dislocation spends time $t_{w}=v_{1}^{-1}$ waiting to get a successful thermal assist, then the average dislocation velocity can be approximately written as,

$$
v_{d} \approx \frac{\lambda}{t_{w}}=\lambda v_{1}=\lambda v_{0} e^{-\Delta F / k T}
$$

A constitutive model should describe the rate of deformation, ie, the strain rate $\mathrm{d} \varepsilon / \mathrm{dt}$, under given conditions of temperature, applied shear stress, and so on. We start with Orowan's equation, [Hull\&Bacon, 1984] which equates strain rate with the product of mobile dislocation density, $\rho_{\mathrm{m}}\left(\mathrm{cm}^{-2}\right)$, discrete step size (Burgers vector), and average dislocation velocity, $\mathrm{v}_{\mathrm{d}}$,

$$
\dot{\varepsilon}=\rho_{m} b \bar{v}_{d}
$$

Combining equations 2 and 3 , and rearranging gives

$$
\dot{\varepsilon}=\rho_{m} b \lambda v_{0} e^{-\Delta F / T}=\frac{\rho_{m} b^{2}}{\frac{b}{\lambda v_{0}} e^{\Delta F / k T}}
$$

However, the activation energy depends on barrier height, area, and shape and in the MTS model [Follansbee, 1986, 1991] is parameterized as 


$$
\Delta F=\Delta F_{0}\left[1-\left(\frac{\sigma}{\sigma_{0}}\right)^{p}\right]^{q}
$$

where $p$ and $q$ parameterize the barrier shape. Combining (4) and (5) gives

$$
\dot{\varepsilon}=\frac{\rho_{m} b^{2}}{\frac{b}{\lambda v_{0}} e^{\frac{\Delta F_{0}}{k T}\left[1-\left(\frac{\sigma}{\sigma_{0}}\right)^{q}\right]^{q}}}
$$

If the applied stress exceeds the Peierls barrier, the dislocations are no longer pinned, but rather, can glide freely, resisted only by phonon drag. In this freely gliding regime, assume that the phonon drag coefficient is $\mathrm{D}$, and equate the force applied per unit area to the dislocation with the drag force (per unit area) to calculate the steady-state velocity of the dislocation,

$$
\sigma \mathrm{b}=\mathrm{D} \mathrm{v}_{\mathrm{d}}
$$

Combining combining (6) and (7) gives

$$
\dot{\varepsilon}=\frac{\rho_{m} b^{2}}{\frac{b}{\lambda v_{0}}\left\{e^{\frac{\Delta F_{0}}{k T}\left[1\left(-\frac{\sigma}{\sigma_{0}}\right)^{p}\right]^{q}}\right\}+\frac{D}{\sigma}}
$$

which is similar in form to a greatly simplified MTS model [Follansbee, 1986, 1991] or the Steinberg-Lund constitutive equation (if $p=q=1$ ). [Steinberg, 1989] Since the strain rates and shear stresses to be considered in this paper will be very high, we make one final modification to prevent dislocations from moving at or above the shear wave speed, by writing $1 / \mathrm{v}_{\text {eff }}=1 / \mathrm{v}_{\mathrm{d}}+1 / \mathrm{c}_{\mathrm{sh}}$. When this is included, we arrive at 


$$
\dot{\varepsilon}=\frac{\rho_{m} b^{2}}{\frac{b}{\lambda v_{0}}\left\{e^{\left.\frac{\Delta F_{0}}{k T}\left[-\left(\frac{\sigma}{\sigma_{0}}\right)^{p}\right]^{q}\right]}\right\}+\frac{D}{\sigma}+\frac{b}{c_{s h}}}
$$

Equation 9 is given primarily to illustrate mechanisms in high strain rate deformation, as shown by the plot of applied stress versus strain rate in Fig. 1b. In this particular example, at strain rates below $\sim 10^{5} \mathrm{~s}^{-1}$, the applied shear stress is below the Peierls barrier, and the deformation is in the stress assisted thermal activation regime. In this regime, the effect of phonon drag is small, and D could be set to zero with little effect (dashed curve). At strain rates above $\sim 10^{6}$, phonon drag becomes the dominant resistance to deformation, since the applied shear stress exceeds the Peierls stress barrier. Here, the exponential term in the denominator of Eq. 9 can be neglected (dot-dashed curve). Finally, at strain rates $>10^{7}-10^{8} \mathrm{~s}^{-1}$, the barrier set by the shear wave speed limit becomes dominant, and the flow stress rises sharply. (Physically, in this situation, either dislocation density would increase or the deformation mechanisms would change, neither effect of which is represented in this highly simplified constitutive equation.) The thermal activation regime has been extensively studied at lower strain rates. But at the high strain rates addressed here, it is very uncertain how deformation proceeds. How deformation scales with pressure is also uncertain, and the topic of ongoing research. A simpleexample is if strength scales with shear modulus, which is thought to increase with pressure, $\sigma \sim \mathrm{G} \sim \mathrm{f}(\mathrm{P})$, then mechanisms can depend on pressure. At a given strain rate, at low pressure, the mechanism might be in the phonon drag regime, whereas at higher pressure (at the same strain rate), the regime may be that of thermal activation.

One widely used model in the high strain rate regime is the constitutive equation by Steinberg-Guinan. [Steinberg, 1980] The basis for this model is the assumption that above some critical strain rate, $\sim 10^{5} \mathrm{~s}^{-1}$, all effects due to strain rate have saturated, and the constitutive model becomes independent of strain rate. This model is approximately a first order Taylor expansion in pressure and temperature, 


$$
\sigma=\sigma_{i}\left[1+\beta\left(\varepsilon_{i}+\varepsilon\right)\right]^{n}\left[1+\left(\frac{G_{P}^{\prime}}{G_{0}}\right) \frac{P}{\eta^{1 / 3}}+\left(\frac{G_{T}^{\prime}}{G_{0}}\right)(T-300)\right]
$$

where $\eta=\rho / \rho_{0}$ is the compression, $G_{P}^{\prime}=\partial G / \partial P$, and $G_{T}^{\prime}=\partial G / \partial T$.

Concluding this section, there appears to be a unique regime of experimental materials science that could be accessed at ultrahigh pressures $(\mathrm{P} \gg 10 \mathrm{GPa})$ and strain rates $(\dot{\varepsilon} \gg$ $10^{5} \mathrm{~s}^{-1}$ ) that would allow models to be tested in their "asymptotic limit". Since several of the models directly account for scaling of the dynamics with pressure, it would be very valuable to be able to experimentally scale pressure over factors of at least 10 , preferably 100. Finally, the short time scales may make it possible to directly calculate the experimental conditions with MD simulations as well as continuum simulations, and thereby have a rigorous test of constitutive models at the very highest strain rates.

\section{High pressure, high strain rate drivers}

In this section, we show the results of two new experimental techniques for generating a high pressure "drive" to compress samples in the solid state at high strain rate. Each technique has been experimentally demonstrated at pressures up to $\sim 100 \mathrm{GPa}$. Scaling simulations show that on future facilities, this technique should be able to drive samples to much higher pressures in the solid state $(\mathrm{P}>1000 \mathrm{GPa})$.

We show in Fig. 2 the results from a new shockless drive [Edwards, 2003] that we have developed on the Omega laser. [Boehly, 1995] The target consists of a low-Z (typically, $\mathrm{CH}$ ) reservoir of nominal thickness $0.2 \mathrm{~mm}$, followed by a $0.4 \mathrm{~mm}$ vacuum gap, then an Al sample, as illustrated in Fig. 1a. A laser pulse of energy $0.5-1.0 \mathrm{~kJ}$ in a temporally square pulse of duration $4-11$ ns is used to drive a strong shock through the low-Z reservoir. When the shock reaches the back side (the side opposite where the laser was incident), the reservoir unloads into vacuum as an ideal gas in a release wave, as illustrated schematically in Fig. 2b. The pressure wave that is driven into the sample results from the increasing ram pressure, $P_{\text {ram }}=\rho_{\text {ejecta }} v_{\text {ejecta }}^{2}$. This applied pressure increases smoothly and monotonically in time as the reservoir unloads, until the reservoir material 
is depleted. This technique of generating shocklesss compression was modeled after the early work of Barnes using HE as the source of the shock in the reservoir. [Barnes, 1974]

This pressure wave is measured with a line VISAR viewing the back side of a 5-30 $\mu \mathrm{m}$ flat $\mathrm{Al}$ sample, an example image of which is shown in Fig. 2c. The horizontal direction of the image is the "streak" or time direction, and the vertical corresponds to the transverse position along the sample. The interference fringes in the VISAR diagnostic are proportional to velocity, with each fringe shift, $\delta$ (fringe position), corresponding to a known velocity increment, $\delta \mathrm{v}$. Thus, measuring the fringe shift versus time and position on the foil is a direct measure of the velocity of that location of the back side (free surface) of the foil versus time. Several reverberations vs. time are apparent in the image shown in Fig. 2c, but there is no shock discontinuities observable, meaning that the rising pressure pulse is shockless. The reverberations result from the compression waves bouncing back and forth across the $\sim 30 \mu \mathrm{m}$ thick $\mathrm{Al}$ sample, while the reservoir is still unloading.

Using the standard hydrodynamic equations, with a known equation of state of $\mathrm{Al}$, allows the free surface velocity profiles to be back integrated to infer the applied pressure vs. time at the front surface of the $\mathrm{Al}$ sample. The results of such an analysis for four different Omega laser experiments are illustrated in Fig. 2d, showing that as the peak pressure increases, the rise time of the pressure pulse decreases, hence the strain rate of the sample increases. The three solid curves used a carbon resorcinol foam reservoir of initial density $\rho_{\text {reservoir }}=0.1 \mathrm{~g} / \mathrm{cm}^{3}$. The dashed curve corresponds to an experiment where the reservoir was solid density $\mathrm{CH}$ plastic, with $\rho_{\text {reservoir }}=1.0 \mathrm{~g} / \mathrm{cm}^{3}$. Referring to the sketch in Fig 1b, it is easy to see that the strain rate should depend on how fast the reservoir unloads at shock breakout and how large a gap, $\mathrm{L}_{\text {gap }}$, it has to sweep across. The unloading speed is set by the sound speed in the shocked reservoir at shock breakout, $\mathrm{c}_{\mathrm{s}}=$ $\left(\gamma \mathrm{P}_{\text {shock }} / \rho *_{\text {reservoir }}\right)^{1 / 2}$. Hence, the strain rate varies monotonically with $\mathrm{c}_{\mathrm{s}} / \mathrm{L}_{\mathrm{gap}}=$ $\left(\gamma \mathrm{P}_{\text {shock }} / \rho *_{\text {reservoir }}\right)^{1 / 2} / L_{\text {gap }}$. An analytic, closed form equation, validated with numerical simulations, has been derived for the ram pressure versus time on the sample, [Edwards, 2003] given by, 


$$
\rho u^{2}=\left\{\rho_{o}\left[1-\frac{\gamma-1}{\gamma+1}\left(1+\frac{x_{o}-u_{P} t}{c_{o} t}\right)\right]^{\frac{2}{\gamma-1}}\right]\left\{c_{o}\left(\frac{2}{\gamma+1}\right)\left[1+\frac{x_{o}}{c_{o} t}+\frac{\gamma-1}{2} \frac{u_{P}}{c_{o}}\right]\right\}^{2}
$$

The solid curves show the $P_{\text {shock }}-L_{\text {gap }}$ dependence of this drive, where the initial reservoir density was held fixed at $0.1 \mathrm{~g} / \mathrm{cm}^{3}$. The dashed curve shows the result of increasing the initial reservoir density by a factor of 10 . By the $\mathrm{P}_{\text {shock }}-\mathrm{L}_{\text {gap }}$ scaling shown above, the pressure rise time should increase by a factor of $2-3$, which it does. By varying the laser parameters, reservoir density, and gap size in the experiments on the Omega laser, a factor of $\sim 10$ span in strain rate, $5 \times 10^{6} \mathrm{~s}^{-1}$ (curve D) to $5 \times 10^{7} \mathrm{~s}^{-1}$ (curve A) has been achieved with this shockless drive. More recent experiments [Lorenz, 2003] have pushed the peak pressure up to just under 1 Mbar.

The sample compression is nearly isentropic, since there is no shock. This is illustrated by a plot of sample temperature, T versus compression, $\mu=\rho / \rho_{0}-1$, in the inset of Fig. 2d. The data points labeled A, B, .., E correspond to hydrodynamics simulations using the $P(t)$ shown in the main portion of this figure. For comparison, the solid curve represents the room temperature isentrope. At the pressures studied so far in our experiments at the Omega laser, the sample density-temperature conditions are essentially indistinguishable from the room temperature isentrope.

In Fig. 3 we show an alternate approach to achieve high pressures and strain rates in the solid state using staged shocks. In this experiment, done on the Nova laser, $\sim 14 \mathrm{~kJ}$ of laser energy was focused into a $\sim 5 \mathrm{~mm}$ x $3 \mathrm{~mm}$ Au cylindrical radiation cavity (hohlraum) to create a Planckian radiation drive, as illustrated in Fig. 3a. [Kalantar, 2000] The sample was mounted over a hole in the wall of the hohlraum at the midplane. The 20 $\mu \mathrm{m} \mathrm{Al}$ sample was mounted behind $20 \mu \mathrm{m}$ of $\mathrm{CH}(2 \% \mathrm{Br})$ ablator, which converted the radiation flux into ablation pressure. The laser pulse power history is shown in Fig. 3b, and corresponds to a $0.8 \mathrm{TW}, 4 \mathrm{~ns}$ "foot", followed by an intermediate step, then a peak of $\sim 3$ TW for a duration of 3 ns. Simulated temporal profiles for density, pressure, melt 
temperature, and temperature in the $\mathrm{Al}$ near the $\mathrm{CH}(\mathrm{Br})$ - $\mathrm{Al}$ interface are shown in Fig. $3 \mathrm{~b}$. The foot in the laser pulse launches an initial $300 \mathrm{kbar}$ shock that generates a $25 \%$ compression wave in the $\mathrm{Al}$, followed by a second shock driven by the peak of the drive, reaching 1.6 Mbar, at a compression of $\rho / \rho_{0} \sim 1.8$. The laser pulse turns off at $8.5 \mathrm{~ns}$, after which the pressure in the Al decays away in a series of reverberation waves. The resulting strain vs time in the $\mathrm{Al}$ from the simulations is shown in Fig. 3d, reaching a maximum by $20 \mathrm{~ns}$ of 0.9 . During the peak of the drive, the average strain rate was about $7 \times 10^{7} \mathrm{~s}^{-1}$, falling to about $3 \times 10^{7} \mathrm{~s}^{-1}$ later in the drive. This experiment, done on the Nova laser (now shut down), reached a higher peak pressure than the current experiments at the Omega laser, but used $14 \mathrm{~kJ}$ of energy (compared to $<1 \mathrm{~kJ}$ ) and was accomplished with an ablatively driven staged shock drive, as opposed to a ram-pressure driven shockless drive.

We have compiled the $P-\dot{\varepsilon}$ rate conditions accessed in all our materials science experiments conducted so far on the plot shown in Fig. 4. The solid squares show the results of the Omega shockless drive experiments. The open squares represent the corresponding LASNEX simulations of these experiments. The one colored box is a tentative result from a recent experiment, showing that pressures approaching $1 \mathrm{Mbar}$ have been achieved with the shockless drive on Omega. The solid circle corresponds to the staged shock experiment done on the Nova laser. This characteristic $P-\dot{\varepsilon}$ trianglar shape reflects the scaling discussed above, where pressure in the sample is directly proportional to the shock pressure in the reservoir, and strain rate increases monotonically with $c_{s} / L_{\text {gap }}=\left(\gamma \mathrm{P}_{\text {shock }} / \rho *_{\text {reservoir }}\right)^{1 / 2} / L_{\text {gap }}$. Hence, other parameters held constant, strain rate increases monotonically with pressure.

\section{Deformation mechanisms at high strain rate}

In this section we discuss our implementation and use of sample recovery to infer deformation mechanisms, and integral quantities about the drive. In Figs. 5a-c, we show a time sequence from the $2 \mathrm{D}$ hydro code CALE of the crater formation process.

[McNaney, these proceedings, 2003] The simulation assumed a shockless drive similar to those shown in Fig. 2d, with a peak pressure of $50 \mathrm{GPa}$. The time sequence of density 
contours shows that even though the drive pressure pulse turns off by $\sim 30 \mathrm{~ns}$, the crater evolution continues. Note that by $\mathrm{t}=1 \mu \mathrm{s}$, the compression wave has exited the back, and an incipient spall layer appears in Fig. 5c. We show the experimental results in Fig. 5d. of the crater in a recovered large-grained Al6061 sample driven by the shockless drive discussed above, only with a peak pressure of $38 \mathrm{GPa}$. The Al sample has been sectioned and etched to show the crater shape and grain structure of the metal. The overall crater depth is about $300 \mu \mathrm{m}$, in agreement with simulations [McNaney, these proceedings, 2003] and there is structure evident in its shape. The details of the shape of the crater are still being analyzed, but the maximum depth of the crater appears to be a reasonable measure of the integrated drive pulse. For comparison, the crater shown in Fig. 5e was from direct laser illumination with $150 \mathrm{~J}$ in a $\sim 600 \mu \mathrm{m}$ spot for $1 \mathrm{~ns}$. The crater shape is different, and there are suggestions of micro-void formation under the deepest part of the crater, where the launched shock was strongest. There is also evidence of at least one, possibly two incipient spall layers on the back side. Somewhat coincidentally, this spall layer resembles that of the simulation shown in Fig. 5c. If the drive (applied pressure versus position and time) is known, then the crater depth can serve as an integral measure of material strength. This is illustrated in Fig. 5f, where crater depth vs. material strength is plotted from the $2 \mathrm{D}$ simulations. If, for example, the strength of the sample is changed by a factor of two, the crater size is predicted to change by about $50 \%$, which should be readily apparent. Also, our initial inspection of the crater shown in Fig. 5d shows no obvious indication of a melt layer. We also have never seen any evidence of adiabatic shear bands in any of our recovered samples. It may take much larger strains to "turn on" shear bands in our laser experiments. These are issues we will examine in subsequent experiments.

To illustrate the use of recovery to infer deformation mechanism, we show in Fig. 6 the results of recovered samples from shock compessed single crystal $\mathrm{Cu}$. [Meyers, 2003; M.S. Schneider, these proceedings, 2003] A 1 mm thick single crystal sample of $\mathrm{Cu}$ was shock compressed along the (001) direction by directly illuminating the sample with $40-400 \mathrm{~J}$ of laser energy in a $1 \mathrm{~ns}$ pulse in a $2.5 \mathrm{~mm}$ diameter spot on the Omega laser. Since the laser pulse duration is short, the strength of the launched shock decays as it 
moves through the sample. The samples were recovered, sectioned, and analyzed by TEM.

The image shown in Fig. 6a shows the residual microstructure resulting from a $\sim 12 \mathrm{GPa}$ shock, and the image in Fig.6b corresponds to a $~ 40$ GPa shock. There is a very distinct difference in the residual microstructure. The dislocation cell structure shown in Fig. 6a corresponds to the residual tangled dislocations that result from shock deformation due to slip along the 12 dominant slip systems: four $\{111\}$ planes and three $<110>$ slip directions within each of these planes. It is generally assumed that the shock front generates a homogeneous distribution of dislocations to accommodate the shock deformation. After shock passage, the dislocations organize or migrate into this cell structure corresponding to dense dislocation tangles in cell walls, and cell interiors of diameter $d_{\text {cell }}=0.3-0.6 \mu \mathrm{m}$ exhibiting significantly lower residual dislocation density. This post shock migration is driven by the attractive and repulsive forces between dislocations (the so-called Peach-Kohler force). We can make an order of magnitude estimate of the time interval to form this residual dislocation cell structure. Assume that the cells are formed on a characteristic time scale set by the ratio of cell size and characteristic dislocation velocity, and assume that dislocations move at $20 \%$ of the shear wave velocity. Then $t_{\text {cell }} \sim \mathrm{d}_{\text {cell }} /\left(\mathrm{v}_{\text {disloc }}\right) \sim \mathrm{d}_{\text {cell }} /\left(0.2 \mathrm{c}_{\text {shear }}\right) \sim(4 \mu \mathrm{m}) /\left[0.2(\mathrm{G} / \mathrm{\rho})^{1 / 2}\right] \sim(4 \mu \mathrm{m}) /(2$ $\mu \mathrm{m} / \mathrm{ns}) \sim 2 \mathrm{~ns}$. This suggests that these cells should form on a ns time scale after shock passage.

The residual microstructure shown in Fig. $6 \mathrm{~b}$ is considerably different from that shown in Fig. 6a. This image is the result of a TEM analysis with an electron beam direction of $B$ $=<001\rangle$, and the (electron) diffraction plane corresponds to (200). The distinct crosshatch pattern represents traces of $\{111\}$ planes on (001), that is, the edge-on view of the four $\{111\}$ planes cutting the (001) plane. The different hues in the criss-cross pattern represents stacking fault bundles or regions of micro-twins. All four stacking fault variants, (111) $\frac{1}{6}[\overline{1} \overline{1} 2],(11 \overline{1}) \frac{1}{6}[112],(\overline{1} 11) \frac{1}{6}[1 \overline{1} 2],(1 \overline{1} 1) \frac{1}{6}[1 \overline{1} 2]$, are observed (that is, a displacement of $\frac{1}{6}\left[\begin{array}{l}1 \\ 1\end{array} 2\right]$ along the (111) slip plane, and so on) and are indicated by $A, B$, $\mathrm{C}$, and $\mathrm{D}$ in the figure. Given that the laser-induced shock direction was $<001>$, all four 
$\{111\}$ primary slip planes should be activated with equal probability, having the same Schmid factor of 0.4082. [M. Schneider, these proceedings, 2003]

Similar results, both for dislocation cells and for twinning, have been observed by Murr using shocks launched with an HE drive. [Murr, 1981] The "dwell time" for these shocks (the time interval over which pressure is maintained behind the shock) is of order $\sim 1 \mu \mathrm{s}$, whereas for the laser induced shock the dwell time is of order $10 \mathrm{~ns}$ or less, that is, a factor of 100 shorter. This suggests that the observed residual microstructures shown in Figs. $6 \mathrm{a}$ and $6 \mathrm{~b}$ were formed promptly at the shock front. Hence, that the same shockinduced deformation dynamics can be studied on laser-driven systems, where timeresolved diagnostics can be implemented to diagnose the dynamics as it happens, with sub-ns time resolution.

The comparison between the residual dislocation cells shown in Fig. 6a and the microtwins shown in Fig. $6 \mathrm{~b}$ is interesting, because it suggests a shock threshold between 12 $\mathrm{GPa}$ and $40 \mathrm{GPa}$. This threshold can be estimated analytically, as shown in Fig. $6 \mathrm{c}$, by assuming that slip and twinning are competing processes. [Meyers, 2003] Empirically it is known that twinning is rather insensitive to temperature and strain rate, whereas slip is not. Therefore, it is expected that at low strain rates (weak shocks), slip should be the dominant mechanism for deformation, whereas at high strain rates (strong shocks), twinning should dominate. Conversely, at low temperature, twinning should dominate, whereas at higher temperatures, slip should take over. The analytic estimate [Meyers, 2003] for $\mathrm{Cu}$ assumes that the critical twinning shock pressure is independent of both strain rate and temperature. The boundary between slip and twinning is then found by equating the flow stresses for each, $\sigma_{\text {twinning }}=\sigma_{\text {slip. }}$. The flow stress for slip is taken from the MTS model, [Follansbee, 1991], modified to include a Hall-Petch term and a work hardening factor. The strain rate at the shock front is estimated by the empirical SwegleGrady relation, $\dot{\varepsilon} \sim \boldsymbol{P}_{s h k}^{4}$. [Swegle, 1983] The results lead to our estimate of the critical shock pressure above which twinning dominates, and below which slip is the preferred deformation mechanism. This is shown for different initial temperatures and grain sizes in Fig. 6c. For room temperature ambient conditions and single crystal samples, our 
estimate gives $P_{t w i n n i n g}=16-17 \mathrm{GPa}$, which is consistent with the observations shown in Figs. 6a and 6b.

It is also possible to look for this slip-twinning shock threshold in molecular dynamics (MD) simulations. This has been done for a shock in single crystal $\mathrm{Cu}$ using the embedded atom method (EAM) with a potential from Mishin. [E. Bringa, these proceedings, 2003] This potential has been fitted to hydrostatic data, ab-initio cold curve results, and elastic constants. The shock simulations compared very favorably with principle Hugoniot data from shocked single crystal $\mathrm{Cu}$. Figure $6 \mathrm{~d}$ shows the results from a MD simulation of a $50 \mathrm{GPa}$ shock in the $<100>$ direction of single crystal $\mathrm{Cu}$. The circles represent individual lattice sites, and the color represents the centro-symmetry parameter. One can see in Fig. 6d regions of stacking faoult bundles, which are the precursor to twinning. Similar simulations run out later in time did indeed show the twinning transition. [E. Bringa, these proceedings, 2003] Note the qualitative similarity between Fig. $6 \mathrm{~d}$ and $6 \mathrm{~b}$, showing that the MD simulations are reproducing the shock dynamics observed in the experiment. At $\mathrm{P}_{\text {shk }}<40 \mathrm{GPa}$, no obvious twinning was observed in the MD simulations, and the deformation was by slip alone. The likely reason that the twinning threshold in the MD simulations is higher than that estimated from the experiments is that the "single crystals" used in the experiments actually contained some level of initial dislocations and defects, whereas the MD simulations do not.

\section{Material Strength at High Pressure and Strain Rate}

To dynamically infer material strength, we are developing hydrodynamic instability experiments, following the technique demonstrated in the early work by Barnes. [Barnes, 1974] By accelerating a metal sample or payload with a lower density, higher pressure "pusher", we create a situation where the interface is hydrodynamically unstable to the Rayleigh-Taylor (RT) instability. Any pre-existing perturbations will attempt to grow, and material strength will act to counter or slow this growth. Therefore, by measuring the RT growth of machined sinusoidal ripples in metal foils that are accelerated by the 
drive, and comparing the observed perturbation growth with that expected for an equivalent liquid target, allows material strength at high pressure and strain rate to be inferred.

One technique to dynamically measure the growth of ripples is with time-resolved faceon radiography. Consider, for example, the experimental configuration shown in Fig. 3a. The metal sample being studied was machined with sinusoidal ripples of wavelengths 10 , 20 , and $50 \mu \mathrm{m}$, then thermally press-fitted onto a $\mathrm{CH}(\mathrm{Br})$ ablator. This target package is mounted over a hole in the wall of a radiation cavity (hohlraum). The radiation deposits its energy in the $\mathrm{CH}(\mathrm{Br})$ at the ablation front, generating an ablation pressure which pushes on the higher density Al-6061, causing it to accelerate. In the accelerating reference frame of the interface, the higher density $\mathrm{Al}$ is "sitting on top of" the lower density $\mathrm{CH}(\mathrm{Br})$ fluid, a configuration which is hydrodynamically (RT) unstable. The tips of the ripples of Al will try to "sink to the bottom", causing the ripple amplitude to grow. Additional laser beams are delayed by $10-21 \mathrm{~ns}$, then focused onto a Sc disk, creating a burst of $4.3 \mathrm{keV} \mathrm{He}-\alpha \mathrm{x}$-rays. These hard X-rays penetrate the foil in-flight, and are recorded in a gated $\mathrm{x}$-ray pinhole camera. [Budil, 1997] As the perturbations at the $\mathrm{CH}(\mathrm{Br})-\mathrm{Al}$ interface grow due to the RT instability, the differential absorption (contrast) of the "backlighter" $\mathrm{x}$-rays is increased. The thick regions of the $\mathrm{Al}$ absorb more than the thin regions, so the growing ripples can be measured with good spatial $(<10 \mu \mathrm{m})$ and time $(<1 / 2 \mathrm{~ns})$ resolution. An example of such an inflight radiograph is shown in Fig. $7 \mathrm{a}$, from an experiment driven with the hohlraum drive shown in Fig. 3. The target was $20 \mu \mathrm{m} \mathrm{CH}(2 \% \mathrm{Br})$ backed by $20 \mu \mathrm{m}$ Al6061-T6. The dark vertical stripes shown in the radiograph in Fig. 7a correspond to the peaks of the ripples (the RT "spikes"), and the bright (white) regions correspond to the valleys (the RT "bubbles"). The pre-imposed sinusoidal ripples had wavelengths of $\lambda=10 \mu \mathrm{m}, 20 \mu \mathrm{m}$ and $50 \mu \mathrm{m}$, and initial peak-tovalley heights of $1 \mu \mathrm{m}$. From analysis of a series of such laser shots done on the Nova laser, plots of perturbation growth factor versus time were assembled, as shown in Figs. $7 \mathrm{~b}-\mathrm{d}$. The squares and the circles correspond to the same data, only analyzed two different ways. Each represents the average over several (5-15) independent data points under nominally identical conditions, and each individual datum was assigned an error 
bar. The squares are a weighted average of the individual points, and the error represents the error bar of the mean. The circles result if each individual data point is given equal statistical weight, and the error bars now represent the standard deviation of the full distribution that went into the average. This representation reflects the level of scatter present in the individual data points. [Kalantar, 2000]

The curves represent 2D simulations with the code LASNEX, using the Steinberg-Guinan constitutive model [Steinberg, 1980] (Eq. 10). The dotted curves assume that the Al was liquid, that is, the material strength in the $\mathrm{Al}$ was set to $\sigma=0$. The solid curve represents the result if $\sigma=\sigma_{S G}$ throughout the simulation, that is, if nominal Steinberg-Guinan strength were used. Looking at all three ripple wavelengths, we conclude that neither of these two calculations satisfactorily reproduces all of the data. The calculation using the nominal Steinberg-Guinan model underpredicts the growth of the $10 \mu \mathrm{m}$ ripple throughout, underpredicts the growth of the $20 \mu \mathrm{m}$ ripple early in time (16 ns), and slightly overpredicts the growth of the $50 \mu \mathrm{m}$ rippled data late in time (21ns). The liquid calculation ( $\sigma=0$ throughout) overpredicts the late time data for all three wavelengths, with the discrepancy the most severe for the $10 \mu \mathrm{m}$ and $20 \mu \mathrm{m}$ ripples. It would appear that a model intermediate between $\sigma=0$ and $\sigma=\sigma_{\text {SG }}$ would better to reproduce the data. To gain some insight, we did an additional simulation where we set $\sigma=0$ for $\mathrm{t}<13 \mathrm{~ns}$, then set $\sigma=\sigma_{\mathrm{SG}}$ thereafter. These simulations (not shown) were very similar to $\sigma=0$ (liquid) simulations shown by the dotted curves. Apparently the material strength required to stop material that was already flowing (from bubble to spike in the RT ripples) requires significantly enhanced strength. The dot-dashed curves corresponds to simulations where we set $\sigma=0$ for $\mathrm{t}<13 \mathrm{~ns}$, then set $\sigma=\mathrm{G}_{\mathrm{SG}} / 10 \approx 5 \sigma_{\mathrm{SG}}$ thereafter. The motivation for setting $\sigma=\mathrm{G} / 10$ was to see what the effect would be if we set the strength to be that of an ideal crystal (no dislocations, no defects) at $\mathrm{t}>13 \mathrm{~ns}$. Interestingly, this ad hoc model does better reproduce the data. Whether there is physical meaning behind this 2-step approach remains to be seen. Possibly at the very high strain rates of this experiment (mid $\times 10^{7} \mathrm{~s}^{-1}$ ), dislocation transport becomes ineffective at accommodating the deformation, leaving whole rows of atoms to be sheared, in response to the high applied shear stress, consistent with setting $\sigma=\mathrm{G} / 10$. Alternatively, looking at $\sigma \sim 5 \sigma_{\mathrm{SG}}$ 
might suggest that at these high strain rates, the dislocations generate very small cells and dense tangles in the cell walls, such as shown for $\mathrm{Cu}$ in Fig. 6a. Recall, our estimate showed that the time scale to form a dislocation cell pattern was only a few ns. These cell walls impede dislocation transport. [Murr, 1981] If the cell size was at least $\sim 25$ times smaller than the initial grain structure, which is very plausible, an effective HallPetch effect, $\sigma \sim\left(d_{\text {cell }}\right)^{1 / 2}$, could lead to the factor of 5 enhancement of strength over the nominal Steinberg-Guinan model.

Closer examination of the radiograph shown in Fig. 7a suggests yet a third possibility. This image shows additional random 3D structure superposed on the $2 \mathrm{D}$ sinusoidal ripples. To consider whether this $3 \mathrm{D}$ random structure could be related to the initial grain structure, we show (in a face-on view) the initial grain structure in Fig. 8a. The grains in the face-on view had sizes of $10-50 \mu \mathrm{m}$, that is, the same size as the preimposed ripples whose RT growth we are measuring. The foils were rolled to $20 \mu \mathrm{m}$ thick, and in this thin (axial) direction, the grains were severely flattened to 1-2 $\mu \mathrm{m}$ thickness. To access whether the random 3D pattern observed is grain related, we did the following analysis. In Fourier space, we filtered out the 2D sinusoidal ripples, then in physical space fit what remains with a pattern recognition algorithm. This is shown in Figs. $8 \mathrm{~b}$ and $8 \mathrm{c}$.

Qualitatively, all three patterns shown in Fig. 8c look rather similar. To quantify this comparison, we take the power spectra of these patterns, as shown in Fig. 8d. The correlation between the random structure in the inflight radiographs with the initial grain structure is not perfect, but close enough to motivate asking what affect the grains might have in this dynamic experiment.

Examination of Fig. 3c shows that the A16061 foil experienced a $30 \mathrm{GPa}$ shock followed by a $160 \mathrm{GPa}$ shock. We carried out an MD simulation to examine what might happen when a $30 \mathrm{GPa}$ shock traverses a grain boundary. The simulation used the EAM method with an Ercolessi-Adams potential. A single $\Sigma 5$ tilt grain boundary was created (Fig. 9a); the grain boundary has a $\sim 25 \%$ lower density than the surrounding medium, due to the dislocations that it contains. A calculation of the effect when a $30 \mathrm{GPa}$ shock traverses the grain boundary was carried out (Fig. 9b). The simulation suggests that the shocked 
grain boundary is hotter by several hundred degrees than the surrounding medium, likely due to its lower density. This raises the possibility that after the second, much stronger shock (160 GPa), the grain boundary could be hot enough to melt, or at least largely loose its strength. [There is a competing process of electron heat conduction that would work to rapidly cool the grain boundary, as the shock heats it. We are currently examining this shock heating vs. conduction cooling competition.]

To assess the effect of grain boundary heating on the RT dynamics, we have started "mesoscale" simulations using the ARES code. We have explicitly coded up grain boundary regions and grain interior regions. We set $\sigma=0$ in the grain boundary regions, to represent liquid grain boundaries, but leave $\sigma=\sigma_{\mathrm{SG}}$ (nominal Steinberg-Guinan strength) elsewhere. The results at $12 \mathrm{~ns}$ are shown in Fig. $9 \mathrm{c}$, where the $20 \mu \mathrm{m}$ ripple is starting to grow. The color corresponds to strength, so the white regions represent $\sigma=0$ at the grain boundaries. Growth factors vs time from ARES are shown in Fig. 9d. The upper red curve corresponds to $\sigma=0$ everywhere, the lower blue curve represents $\sigma=\sigma_{\mathrm{SG}}$ everywhere, and the intermediate black curve represents the case where $\sigma=\sigma_{\mathrm{SG}}$ everywhere except at the grain boundaries (GB), where $\sigma_{\mathrm{GB}}=0$. This mesoscale simulation falls in between the other two, and seems to be a physically plausible explanation for the observed early time loss of strength. In this scenario, if the melted grain boundaries resolidified, the resulting structure might be micro-crystaline with $\sim 100$ $\mathrm{nm}$ grain sizes, or even amorphous. [Meyers, these proceedings, 2003] If the former, the Hall-Petch effect of $\sigma \sim \mathrm{d}_{\text {grain }}{ }^{-1 / 2} \sim 5 \sigma_{\mathrm{SG}}$ suggests enhanced strength, whereas bands of amorphous material might shut off dislocation transport entirely, suggesting $\sigma \sim \mathrm{G} / 10$.

The facility where the data shown in Figs. 3 and 7 were taken, the Nova laser, has been shut down and disassembled. So this exact experiment cannot be reproduced. At this point, we conclude the analysis and interpretation of this past experiment. Which of the above physical scenarios proves to be correct, if any, will await future, more extensive and discriminating data and calculations.

\section{Lattice Response}


We now leave integral experiments, and turn to two exciting new microscale diagnostics to probe the lattice response, time resolved diffraction and EXAFS. These two diagnostics offer enormous possibilities to infer such fundamental quantities as phase, Peierls barrier, dislocation density, and temperature. Experiments done with single crystals are well suited to the time resolved diffraction diagnostic. If a shock or compression wave traverses a single crystal, the lattice planes compress. This can be observed by recording a Bragg diffraction signal, both in reflection and in transmission, as illustrated in Fig. 10a. If a shock is launched from top to bottom in Fig. 10a through, say, a $40 \mu \mathrm{m}$ thick single crystal Si sample, the shock compression can be tracked by streaking the diffraction signal (aligning the diffraction image onto the slit of an $\mathrm{x}$-ray streak camera), as shown by the raw image in Fig. 10b. [Loveridge-Smith, 2001] Initially there is diffraction only off the unshocked region. Then later in time, there are regions of the $\mathrm{Si}$ that have been shocked, and regions that remain unshocked. If the x-ray probe is high enough energy, $\sim 5 \mathrm{keV}$ here, then both regions can be observed simultaneously. A time-integrated image can also be recorded onto x-ray film, as shown in Figs. 10c-f. Figures 10c and 10d correspond to Si and the geometry indicated in Fig. 10a. This experiment was done on the Nova laser using a hohlraum drive, similar to that shown in Fig. 3. Figure 10e and $10 \mathrm{f}$ correspond to a similar experiment done on the Omega laser, now using a single-crystal $\mathrm{Cu}$ sample shocked in the [001] direction by direct laser illumination. [Loveridge-Smith, 2001] Both $\mathrm{Si}$ and $\mathrm{Cu}$ show lattice compression in the reflection Bragg data. The transmission Bragg detector, however, was oriented to diffract off a lattice plane orthogonal to the direction of shock propagation, thus measuring transverse lattice compression (see Fig. 10a). For the shocked Si crystal, there was no observed transverse compression, to within the limits of sensitivity of the diagnostic, whereas for the $\mathrm{Cu}$ sample there was prompt relaxation to a 3D compression (quasi-hydrostatic). So we conclude that the Si crystal was compressed uniaxially over the $10 \mathrm{~ns}$ duration of the shock experiment, whereas the $\mathrm{Cu}$ crystal was compressed nearly hydrostatically. This observation can be interpreted as follows. Relaxation to a 3D compression requires dislocation movement. Orowan's equation, $\dot{\varepsilon}=\rho_{m} b \bar{v}_{d} \quad$ (ie, Eq. 3) states that the rate of deformation, quantified by strain rate $\mathrm{d} \varepsilon / \mathrm{dt}$, 
is equal to the product of the density of mobile dislocations, $\rho_{m}$, the Burgers vector $b$, and the average dislocation velocity, $\mathrm{v}_{\mathrm{d}}$. So the determining factors are the mobile dislocation density and the average dislocation speed. But $\left\langle v_{d}\right\rangle$ is a function of the Peierls barrier, as shown in Eq. 6. At a given T, if $\sigma_{0}$ is high, $\left\langle v_{d}\right\rangle$ and hence $\mathrm{d} \varepsilon / \mathrm{dt}$ is low, because the mobile dislocations stay pinned for long intervals of time. Materials with low mobile dislocation density or high Peierls-Nabarro stress, will have a low de/dt, and the transition to 3D compression will take longer. It is well known, however [Hull and Bacon, 1984] that the Peierls barrier in fcc metals such as $\mathrm{Cu}$ is very low, $\sigma_{0} \sim\left(10^{-6}-10^{-}\right.$ $\left.{ }^{5}\right) \mathrm{G}$, where here $\mathrm{G}$ is the shear modulus. Covalent crystals, on the other hand, have a large barrier, $\sigma_{0} \sim\left(10^{-2}\right) \mathrm{G}$. Silicon has both a large $\sigma_{0}$ and low $\rho_{\mathrm{m}}$. Hence, the time to transition to 3D compression for $\mathrm{Si}$ will be very long, compared to $\mathrm{Cu}$, qualitatively explaining Fig. 10. This data and analysis shows the potential value of time-resolved diffraction for microscopically probing lattice response to infer bounds on Peierls stress and mobile dislocation density in dynamically deforming crystals.

Whereas diffraction probes the long range order of a lattice, extended $\mathrm{x}$-ray absorption fine structure (EXAFS) probes the lattice short range order. The basis for this diagnostic is illustrated in Fig. 11a. When an atom absorbs a high energy x-ray, and ionize, an electron rises into the continuum. The outgoing wave packet (illustrated by the concentric solid circular curves in Fig. 11a) of the free electron scatters off of neighboring atoms (as illustrated by the dashed circular curves), and the outgoing and reflected waves interfere with each other. Since the square of the total electron wave function is what determines the probability of the process, this interference is observed in fine structure in the $\mathrm{x}$-ray absorption just above an edge. For K-edge EXAFS, this is written as

$$
\chi(k)=-\Sigma_{j} \frac{N_{j}}{k R_{j}^{2}} f_{j}(k) \sin \left\{2 k R_{j}+2 \delta(k)+\psi_{j}(k)\right\} e^{-2 \sigma_{j}^{2} k^{2}} e^{-2 R_{j} / \lambda(k)}
$$

where $\chi(k)=\left[\mu(k)-\mu_{0}(k)\right] / \mu_{0}(k)$ is the normalized absorption of $x$-rays of energy $E_{x}$, where 


$$
\frac{\hbar^{2} k^{2}}{2 m_{e}}=E_{x}-E_{K-e d g e}
$$

The summation is over coordination shells, $\mathrm{N}_{\mathrm{j}}$ is the number of electrons in the shell, and $\mathrm{R}_{\mathrm{j}}$ its radius. The factor, $e^{-2 \sigma_{\mathrm{j}}^{2} k^{2}}$ is the Debye-Waller factor, which causes reduction in this coherent interference due to thermal fluctuations, which create incoherence in the scattered waves.

Over the past couple of years, we been developing a time-resolved EXAFS diagnostic at the Omega laser. [Yaakobi, 2003] The experimental setup is shown in Fig. 11b. Two of the Omega beams are used to shock compress a sample of Ti embedded in $\mathrm{CH}$, and the remaining 58 beams implode an ICF capsule. This implosion generates a short ( $100 \mathrm{ps})$ burst of smoothly varying hard $\mathrm{x}$-rays, $\mathrm{I}=\mathrm{I}_{0} \exp \left(-\mathrm{E}_{\mathrm{x}} / \mathrm{T}\right)$, for the EXAFS absorption. Typical values for the implosion $\mathrm{x}$-ray spectrum might be $\mathrm{I}_{0}=2-3 \times 10^{19} \mathrm{keV} / \mathrm{keV}$ and $\mathrm{T}$ $=1.25 \mathrm{keV}$. A measured raw EXAFS absorption spectrum showing the modulations just above the $\mathrm{K}$-edge for room temperature $\mathrm{Ti}$ is shown in Fig. 11c. A typical analysis Fourier transforms $\mathrm{k} \chi(\mathrm{k})$ or $\mathrm{k}^{3} \chi(\mathrm{k})$ to give the electron radial distribution function, [Konningsberger, 1988] shown in Fig. 11d, where the first 3 coordination shells are evident. The simplest analysis selects only the first coordination shell (to be able to ignore the effects of multiple scattering), then converts back to Fourier space, as shown in Fig. 12a, where $k \chi=k\left(\mu-\mu_{0}\right) / \mu_{0}$ is plotted. This is the form that is then fitted with Eq. 12, as shown in Fig. 12a. Fits of the measurements with EXAFS theory, allows the temperature to be deduced through the Debye-Waller factor. In Fig. 12b, the temperature from two different laser experiments are compared through such an EXAFS analysis, showing the sensitivity of this technique to temperature. Note, the modulations die out more quickly at the higher temperatures. This makes sense, because the higher order modulations correspond to the higher harmonics (shorter electron wavelengths) which are more sensitive to the incoherence caused by the thermal fluctations of the positions of the coordination shells, as illustrated in the theory curves in Fig. 12c. In Fig. 12d, the temperature thus determined, for three different laser shots is shown, along with the 
results of simulations vs. the thickness of the $\mathrm{CH}$ preheat shield. The thinner the preheat shield, the higher the expected Ti temperature, as observed, and simulated. The two key points here are (1) the EXAFS as a temperature diagnostic is sensitive down to $\sim 50 \mathrm{meV}$, which is important for materials science experimentsm, with time resolution $<1 / 2 \mathrm{~ns}$, and (2) the temperature of the sample can be modified by varying the thickness of the $\mathrm{CH}$ preheat shield.

\section{New Frontiers}

Up until now, we have described experiments that have been done on existing laser facilities. Roughly speaking, pressures and strain rates achieved correspond to 10-100 GPa and $10^{6}-10^{8} \mathrm{~s}^{-1}$. With the commissioning of the new National Ignition Facility laser at LLNL, [Paisner, 1994] an opportunity presents itself to increase the pressures and compressions of the solids to much higher values. It will be particularly interesting to see, for example, how Peierls barrier, shear modulus, and material strength scale as pressure is increases 100 -fold above $10 \mathrm{GPa}$. In an ambitious design effort, we have examined what pressure - strain rate regimes we think should be accessible on the NIF laser. These are illustrated in the triangular $P-\dot{\varepsilon}$ plot of Fig. 13. Note the similarity to Fig. 4, only at 10x higher peak pressure. The region labeled NEL corresponds to the first 4 beams (of the 192 beam NIF) being turned on for experiments. It appears that with this new facility, a truly unique new genre of ultrahigh pressure-strain rate materials science experiments and research will become possible. It seems very likely that as we experimentally and computationally push into these new regimes of ultrahigh pressure solid-state dynamics, surprises will await us at every juncture.

\section{REFERENCES}

*This work was performed under the auspices of the U.S. Department of Energy by the Lawrence Livermore National Laboratory under Contract No. W-7405-ENG-48.

J.F. Barnes et al, J. App. Phys. 45(2), 727 (1974).

T. H. Boehly, R. S. Craxton, T. H. Hinterman et al., Rev. Sci. Instrum. 66, 930 (1995)

K.S. Budil et al., Rev. Sci. Instrum. 68, 796 (1997). 
P.S. Follansbee and G.T. Gray III, Matls. Sci. and Eng. 138, 23 (1991).

P.S. Follansbee, in L.E. Murr, K.P. Staudhammer, and M.A. Meyers, editors.

Metallurgical applications of shock-wave and high-strain rate phenomena. (M. Dekker, 1986), p. 45.

Harold J. Frost and Michael F. Ashby, Deformation-Mechanism Maps, The Plasticity and Creep of Metals and Ceramics (Pergamon Press, 1982).

John P. Hirth and Jens Lothe, Theory of Dislocations, $2^{\text {nd }}$ Edition (Krieger Publishing Co., 1982).

D. Hull and D.J. Bacon, Introduction to Dislocations, $3^{\text {rd }}$ Edition (ButterworthHeinemann, 1984).

G.R. Johnson and W.H. Cook, Proc. $7^{\text {th }}$ Intern. Symp. Ballistics, Am. Def. Prep. Org. (ADPA), Netherlands, 1983.

G.R. Johnson et al., ASME J. Eng. Mater. Tech. 105, 42 (1983).

D. H. Kalantar et al., Phys. Plas. 7(5), 1999 (2000).

U.F. Kocks, A.S. Argon, and M.F. Ashby, Thermodynamics and Kinetics of Slip (Pergamon Press, 1975).

D.C. Konningsberger and R. Prins, $X$-ray Absorption: Principles, Applications, Techniques of EXAFS, SEXAFS, and XANES (John Wiley \& Sons, 1988).

K.T. Lorenz et al., in preparation for submittal to Phys. Plasmas (2003).

A. Loveridge-Smith et al., Phys. Rev. Lett. 86, 2349 (2001).

J.M. McNaney et al., these proceedings (2003).

M.A. Meyers et al., Acta Mat. 51, 1211 (2003).

Marc A. Meyers, Dynamic Behavior of Materials (John Wiley \& Sons, Inc. 1994).

Marc A. Meyers and Lawrence E. Murr, Shock Waves and High-Strain-Rate Phenomena in Metals: Concepts and Applications (Plenum Press, 1981).

J. A. Paisner, E. M. Campbell, W. J. Hogan, Fusion Technol. 26, 755, (1994).

D.J. Steinberg, S.G. Cochran, and M.W. Guinan, J. Appl. Phys. 51, 1496 ((1980).

D.J. Steinberg and C.M. Lund, J. Appl. Phys. 65, 1528 (1989). 
J.W. Swegle and D.E. Grady, J. Appl. Phys. 58, 941 (1983).

B. Yaakobi et al., J. Optical Soc. America B-Optical Physics. 20, 238 (2003)

F.J. Zerilli and R.W. Armstrong, J. Appl. Phys. 61 61, 1816 (1987); J. Appl. Phys. 68, 915 (1990).

\section{FIGURE CAPTIONS}

Figure 1: (a) Schematic of Peierls barrier pinning an edge dislocation. (b) Illustration of stress vs. strain rate for a generic MTS-like model.

Figure 2: (a) Configuration for the laser-ICE drive. (b) Schematic of the dynamics of the laser ICE drive. (c) Raw VISAR trace for a laser-ICE shot at the Omega laser. (d) Inferred pressure histories for four different laser shots on Omega for the shockless (ICE) drive.

Figure 3: (a) Configuration for an indirect drive (radiation drive) staged-shock drive, using the Nova laser. (b) The required laser power vs. time, showing a foot, intermediate step, and peak. (c) The corresponding density, pressure, melt temperature, and $\mathrm{Al}$ temperature vs. time for this staged-shock drive. (d) The corresponding strain vs. time.

Figure 4: The regimes accessible in solid-state dynamics (P-de/dt space), using the Omega and (now shut off) Nova lasers.

Figure 5 (a-c) Simulation time sequence of crater formation in thick Al6061-T6. (d) Measured crater in Al6061 for the ICE drive and (e) direct laser illumination shock. (f) Simulation prediction of the sensitivity of crater depth vs material strength.

Figure 6: (a) TEM characterized single crystal $\mathrm{Cu}$ foil shocked at the Omega laser. (b) Same, only for a stronger shock. (c) Theoretical slip-twinning threshold prediction. (d) MD simulations showing the slip-twinning transition.

Figure 7: (a) Inflight radiograph of a solid-state RT experiment using thin foils of Al6061. (b-d) Perturbation growth factors vs time for preimposed wavelengths of 50, 20, and $10 \mu \mathrm{m}$.

Figure 8: (a) Initial grain structure (face-on view). (b) Inflight radiographs. (c) pattern corresponding to the initial grain structure, vs. the patterns observed on the inflight radiographs. (d) Power spectra of the same.

Figure 9: (a) Initial $\Sigma 5$ grain boundary in Al. (b) MD simulation of a $30 \mathrm{GPa}$ shock traversing this grain boundary. (c) ARES 3D "mesoscale" simulations, treating the grain 
boundary regions differently than the grain interiors. (d) The predicted perturbation growth factors vs. time for the mesoscale simulations.

Figure 10: (a) Initial configuration for Bragg diffraction experiments. (b) Raw streaked data for Bragg diffraction off shocked Si crystal. (c) Time integrated result for reflection Bragg diffraction for shocked Si. (d) Same only for transmission Bragg diffraction. (e) and (f) same, only for $\mathrm{Cu}$.

Figure 11: (a) Schematic for the fundametal dyamics of EXAFS. (b) Experimental configuration for the EXAFS measurements at the Omega laser. (c) Raw EXAFS absorption spectrum. (d) The inferred electron radial distribution function.

Figure 12: Results from the EXAFS experiments done on the Omega laser to infer temperature of a Ti foil.

Figure 13: Regimes predicted to be accessible for materials science (solid-state) dynamics on the NIF laser. 
(a)

Phonon drag regime

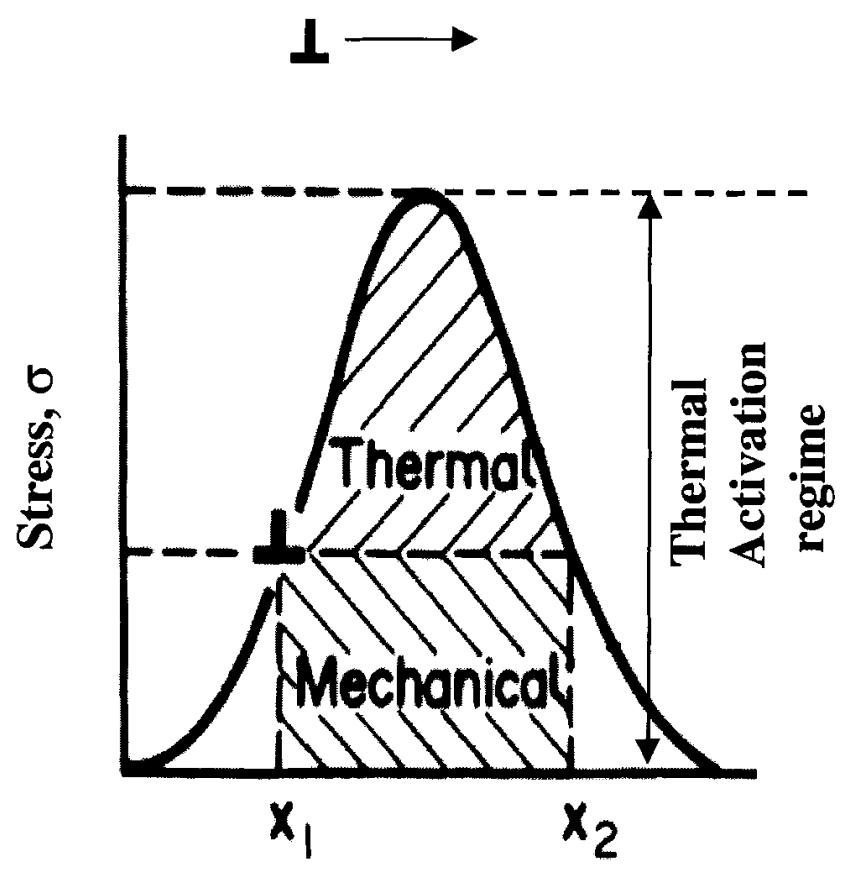

(b)

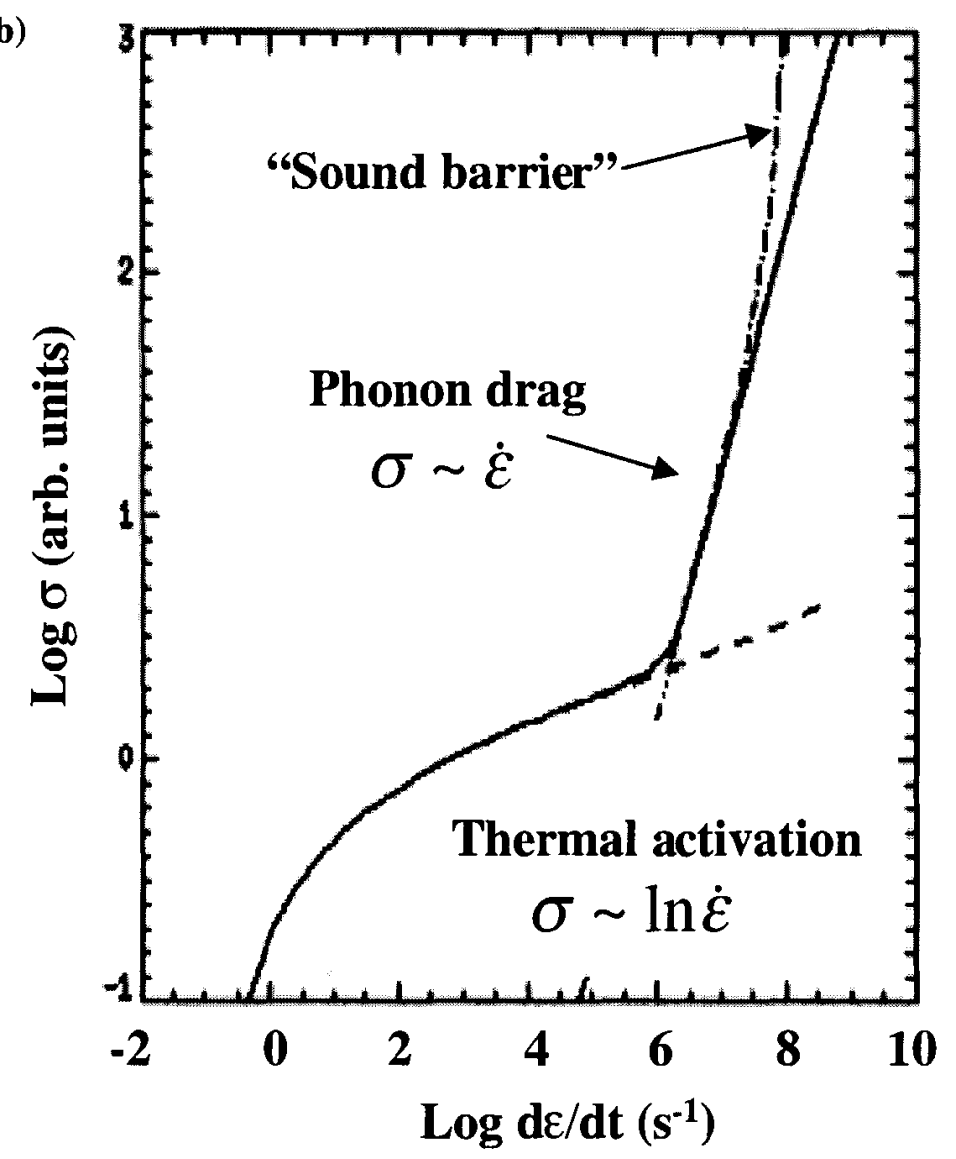


(a)

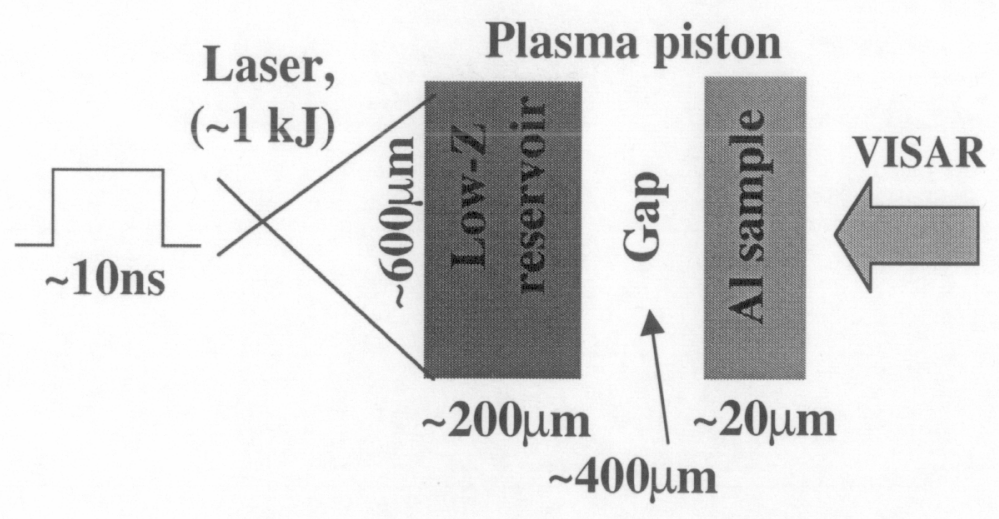

(c)

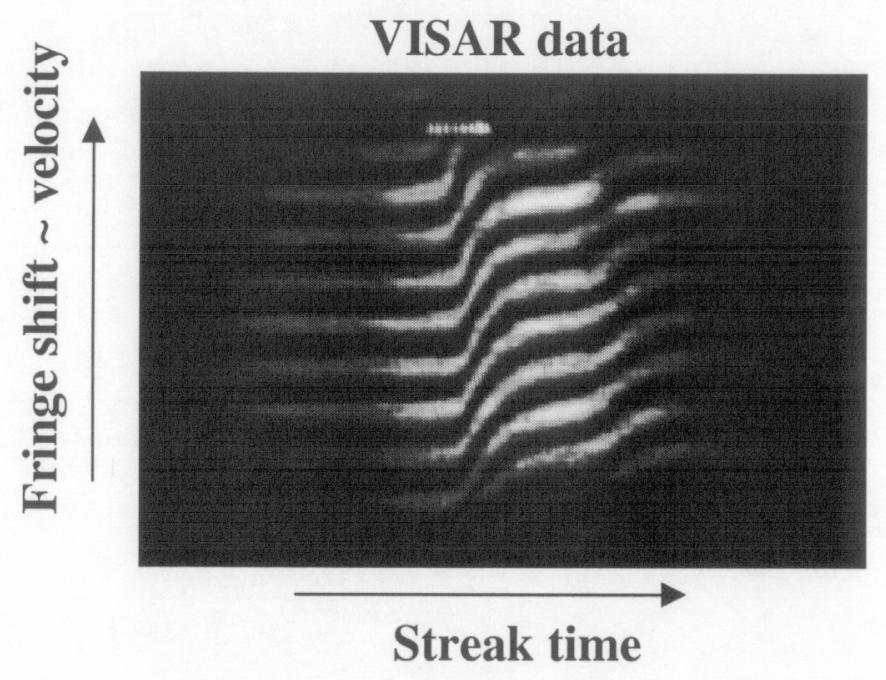

(b)

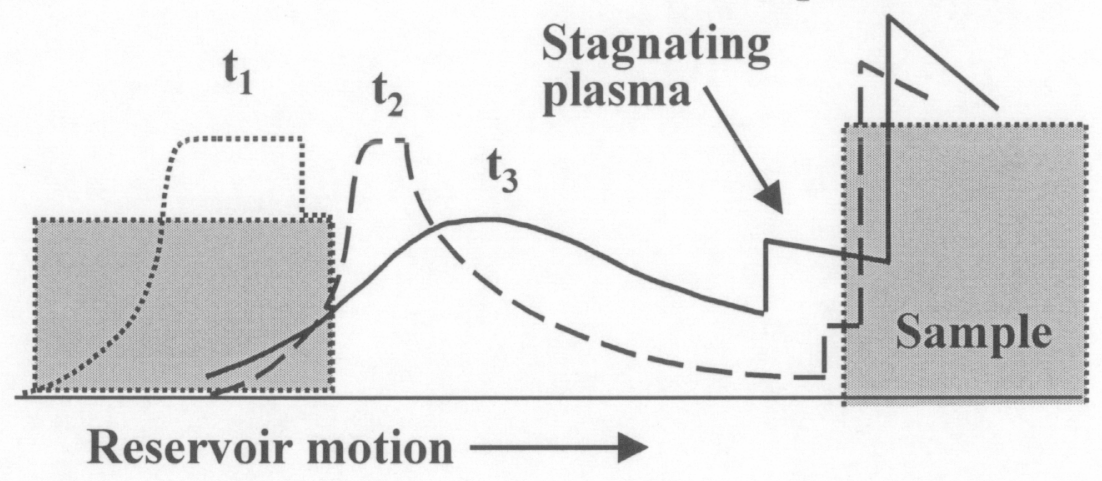

(d) $\mathbf{0 . 6}-1111111111111111111111111111111 \mathrm{l}$

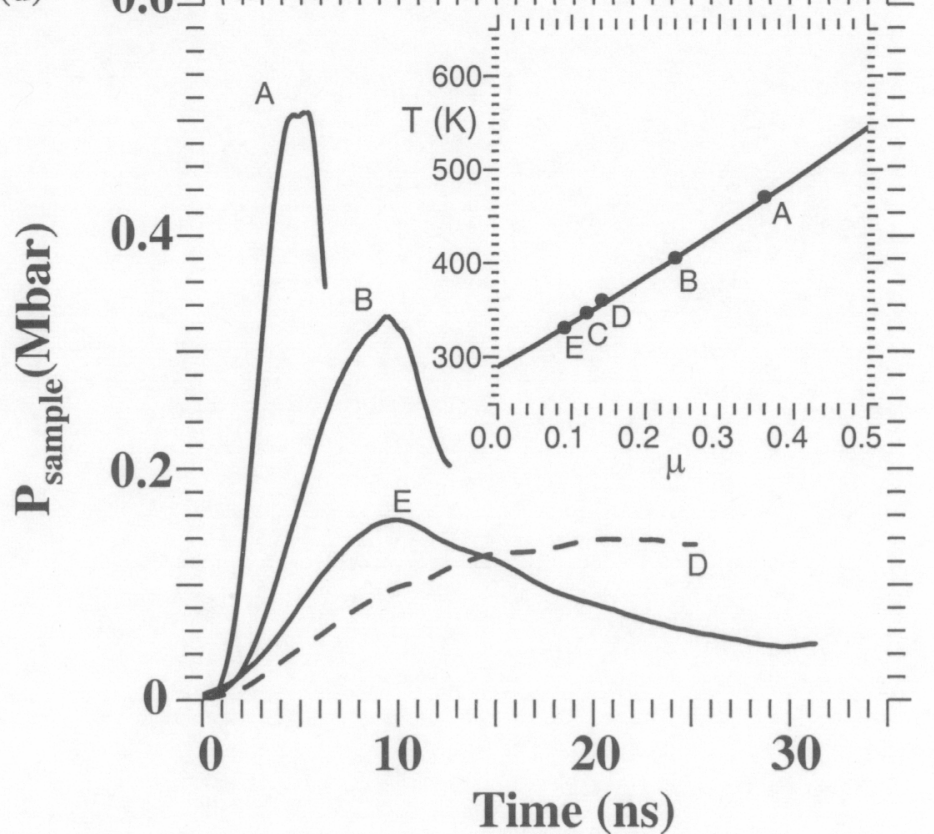

BAR_TMS_paper_figs_3.ppt.2 
(a)
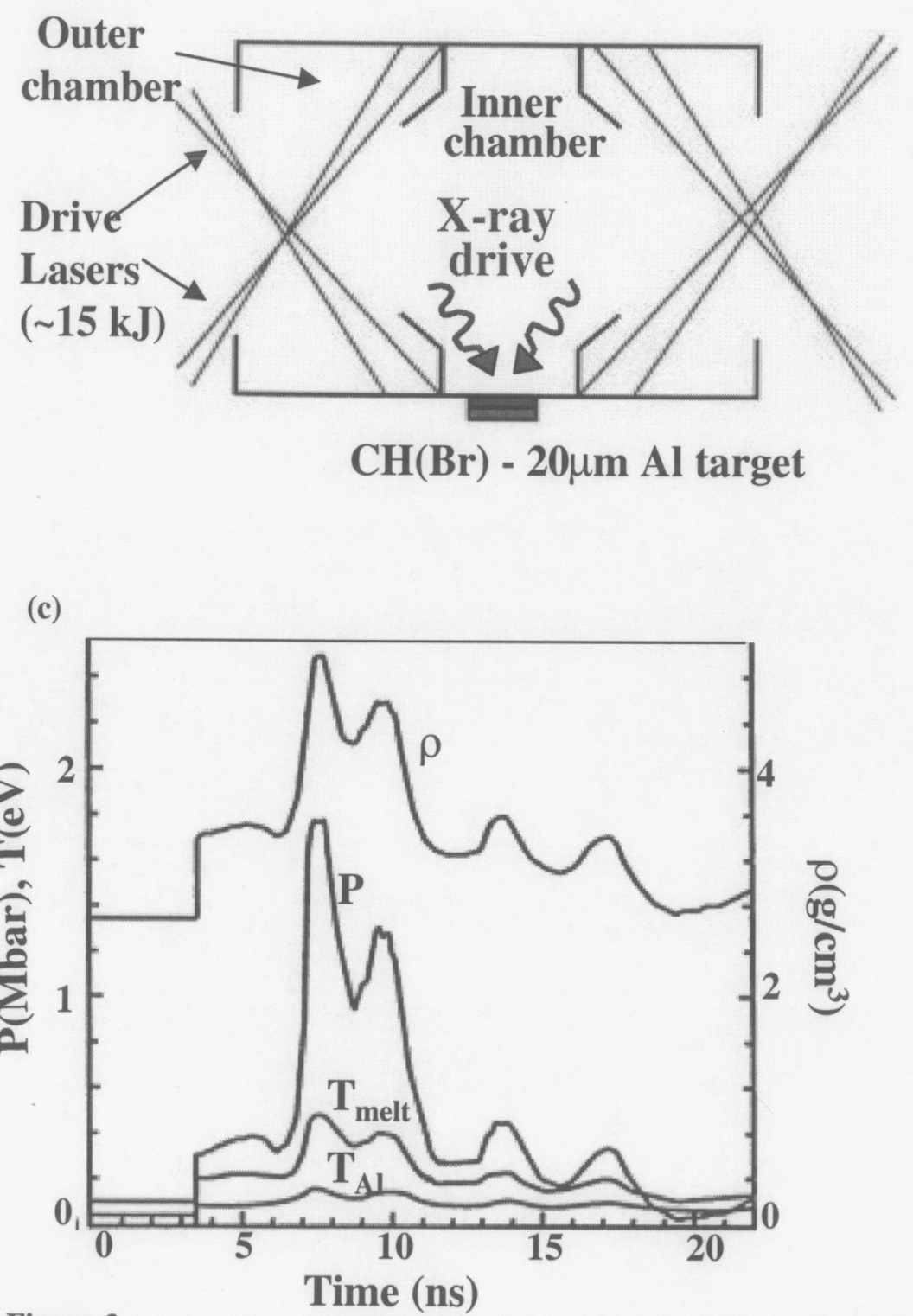

Figure 3 (b)

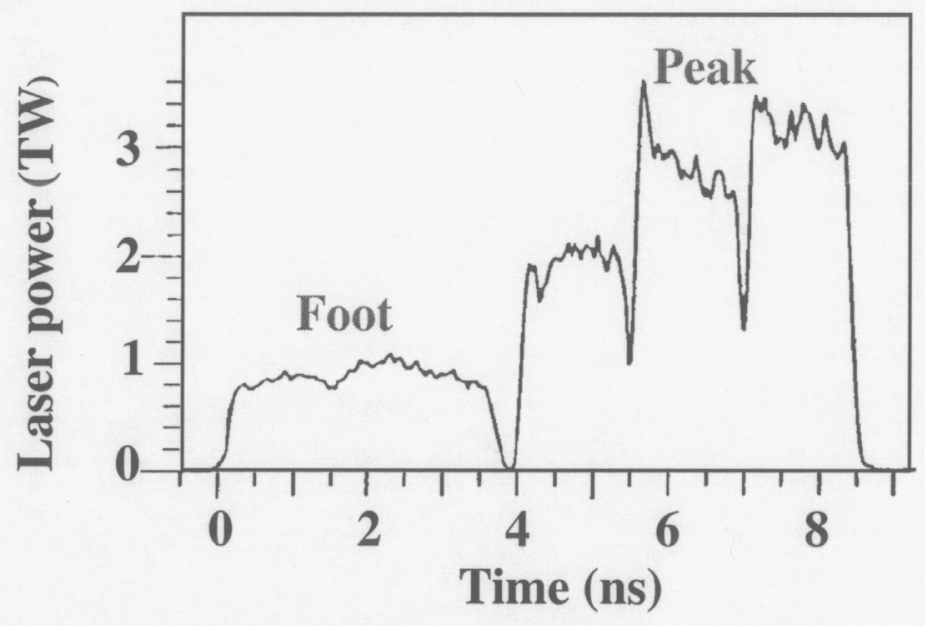

(d)

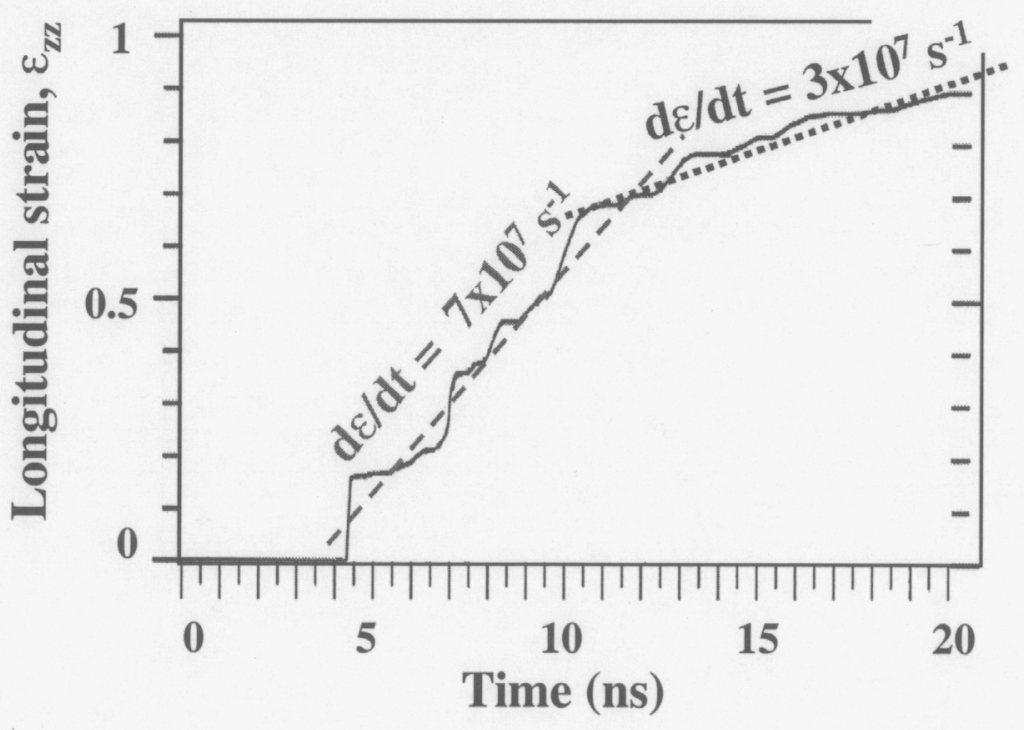

BAR_TMS_paper_figs_3.ppt.3 


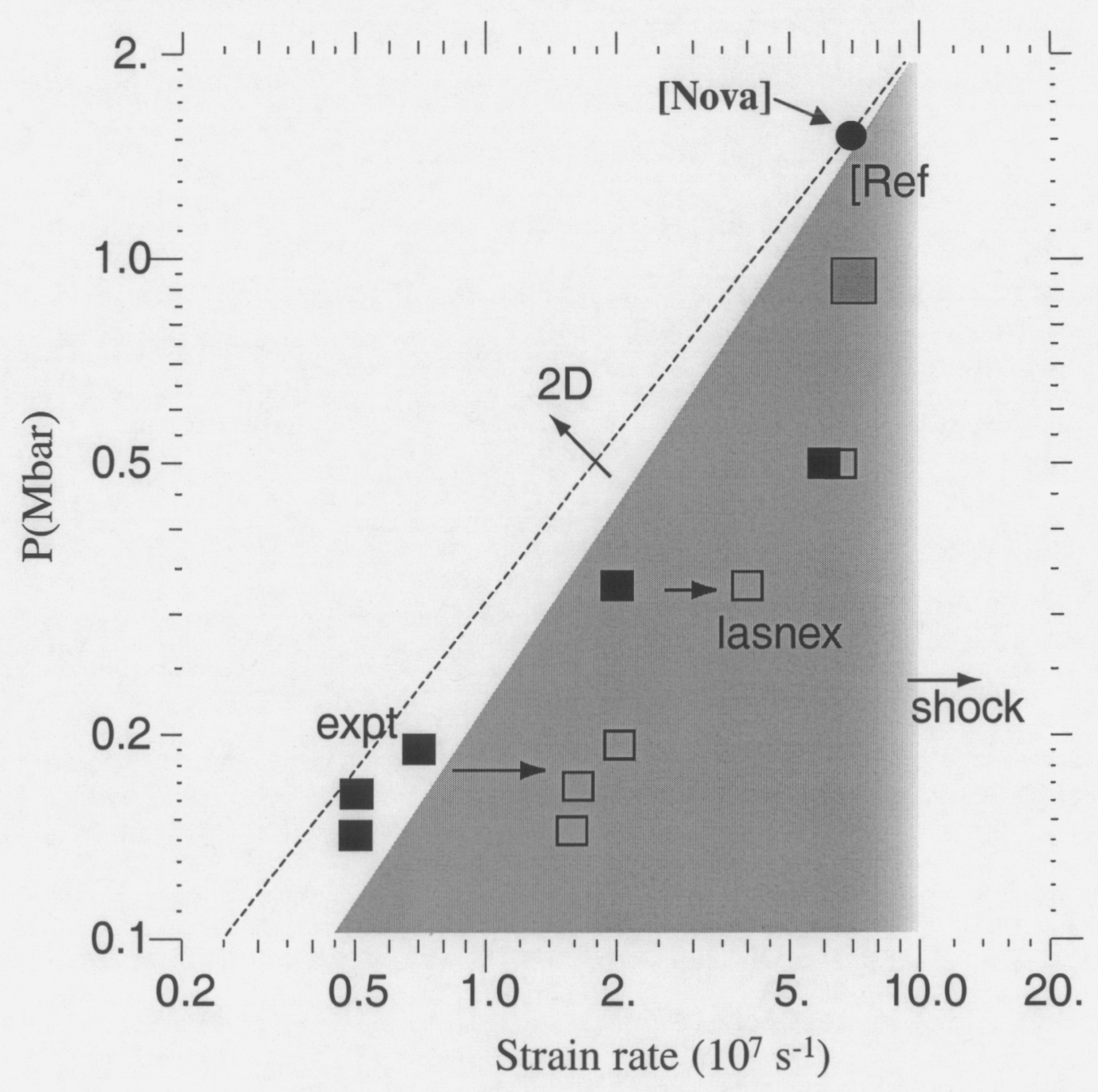

Figure 4 
2D CALE simulation, Al6061 sample, $P_{\max }=50 \mathrm{GPa}$
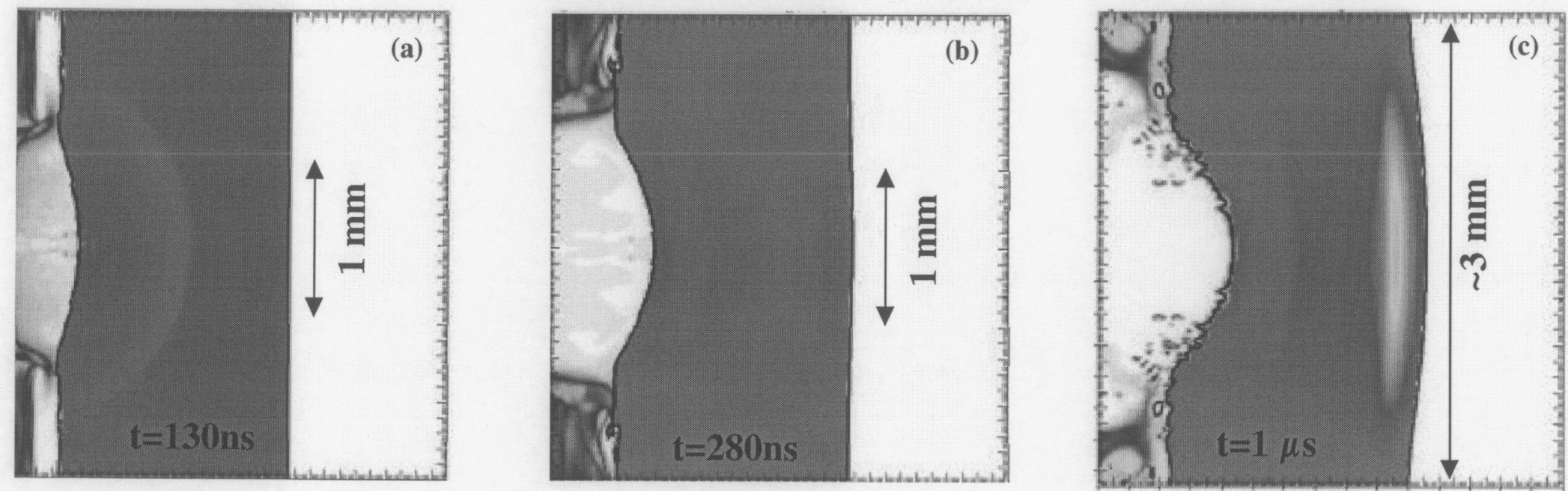

(d)

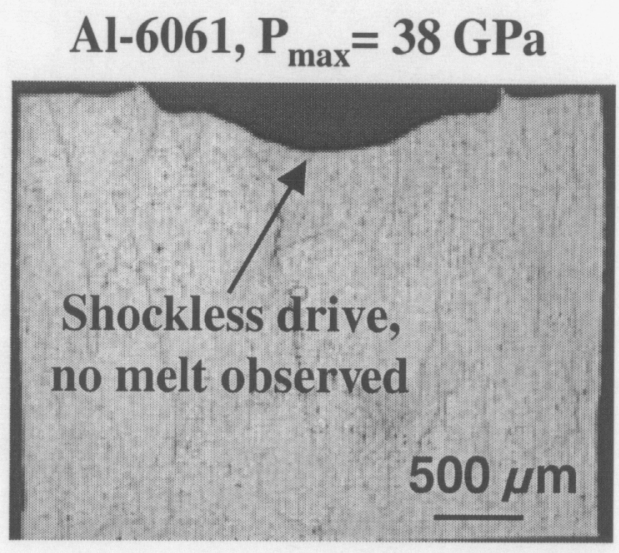

Figure 5

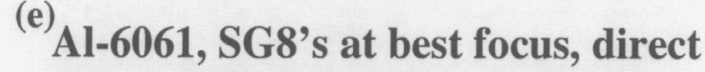
illum. 150J in $1 \mathrm{ns,} \mathrm{Apr.} 2002$ shot

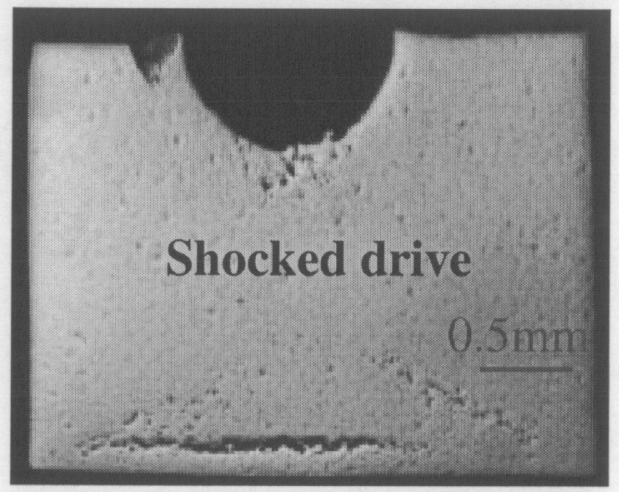

(f)

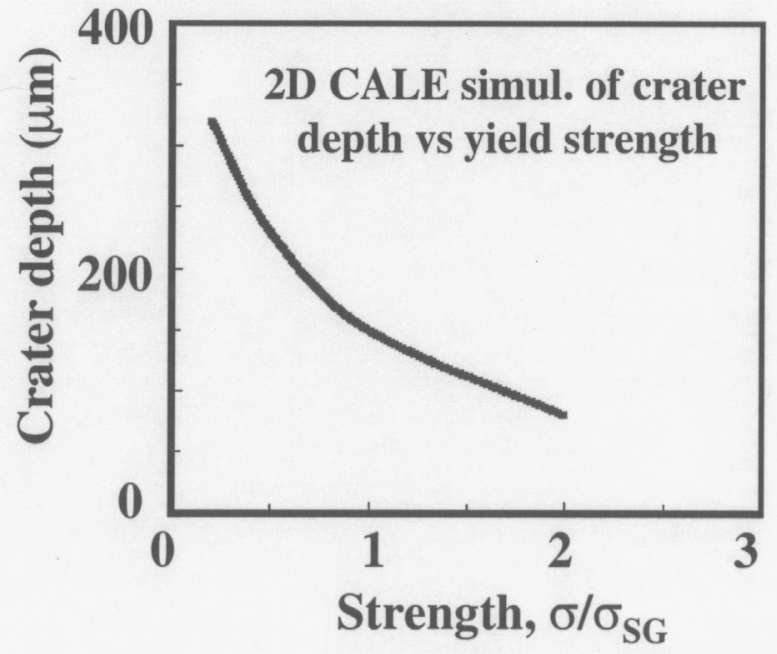


(a)

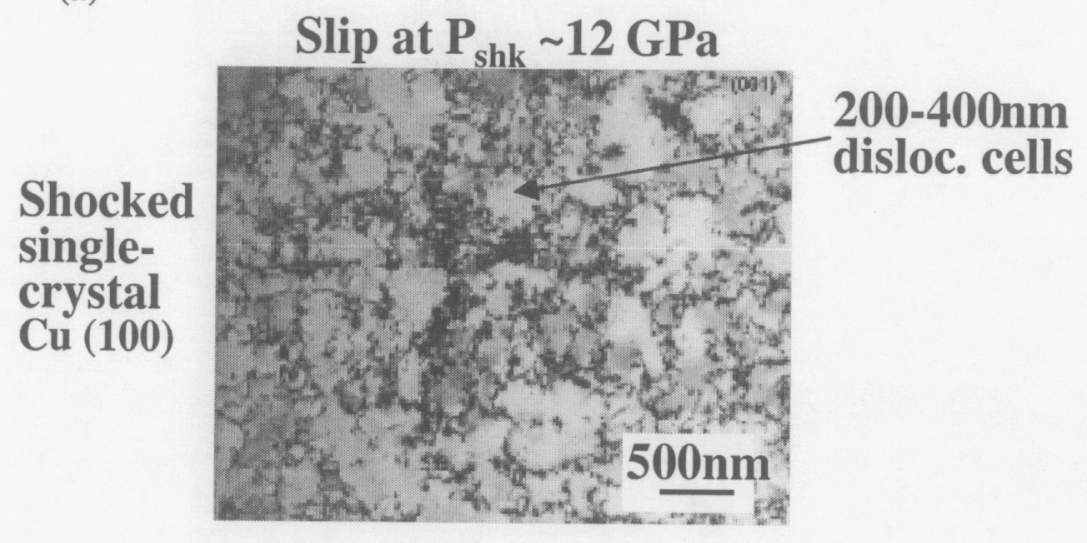

(c)

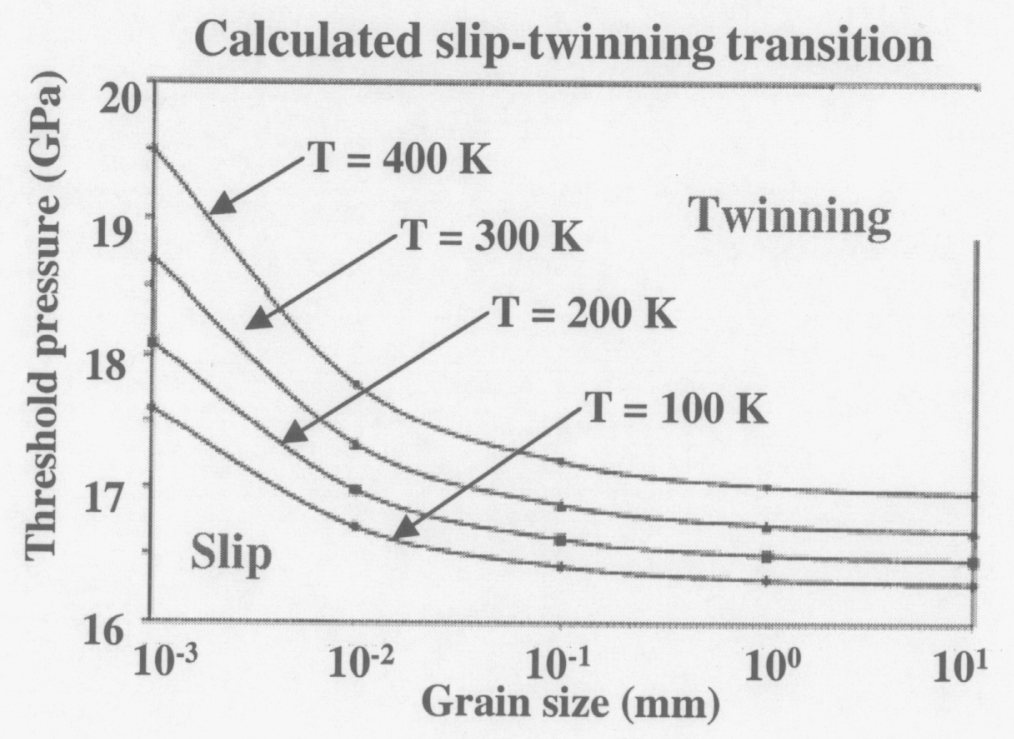

(b)

$$
\text { Twinning at } P_{\text {shk }} \sim 40 \mathrm{GPa}
$$

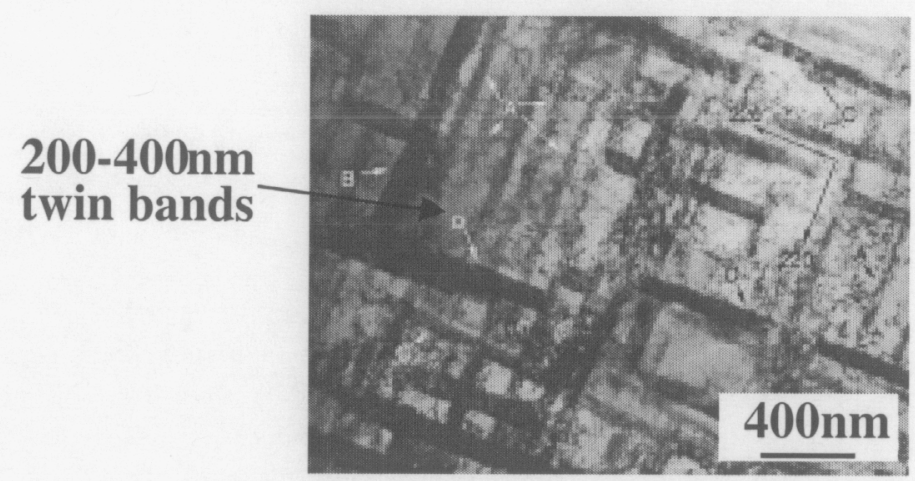

Stacking fault bundles in MD simulations

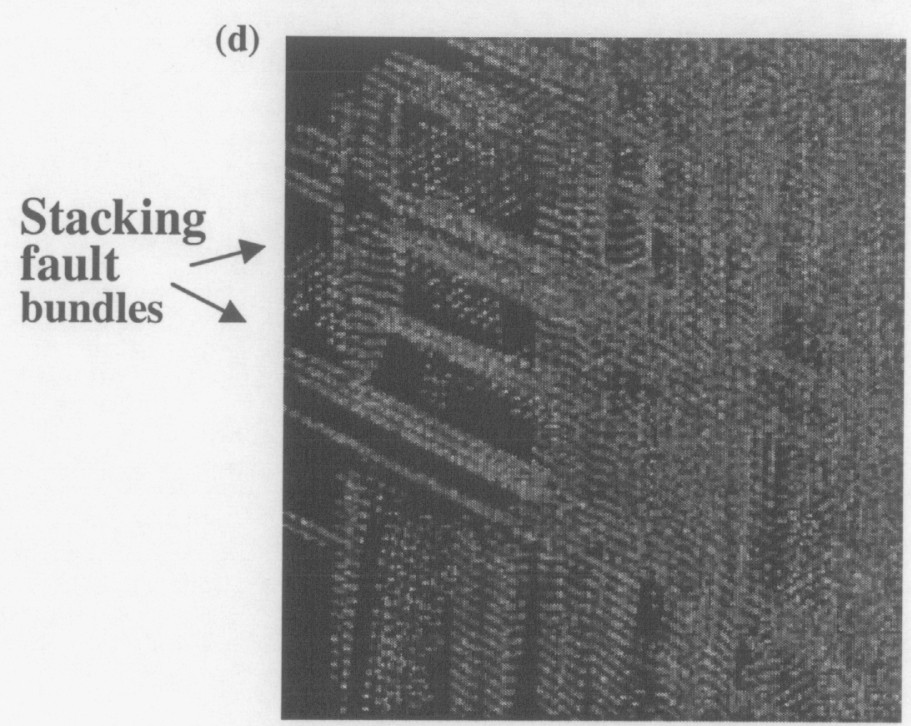

BAR_TMS_paper_figs_3.ppt.6 
(a)

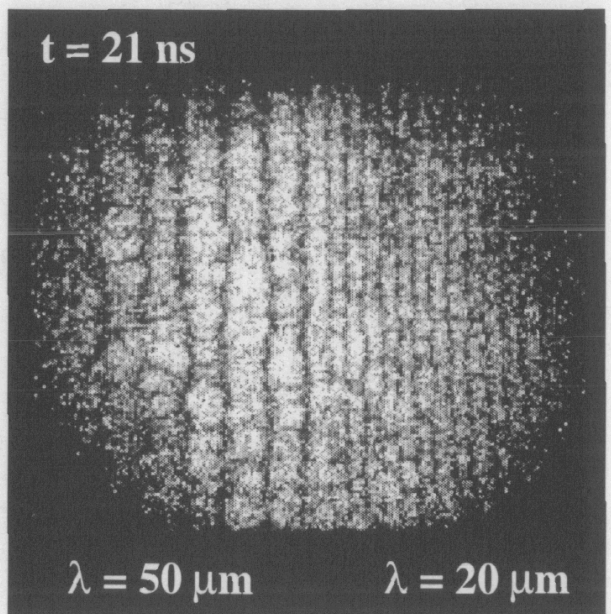

(c)

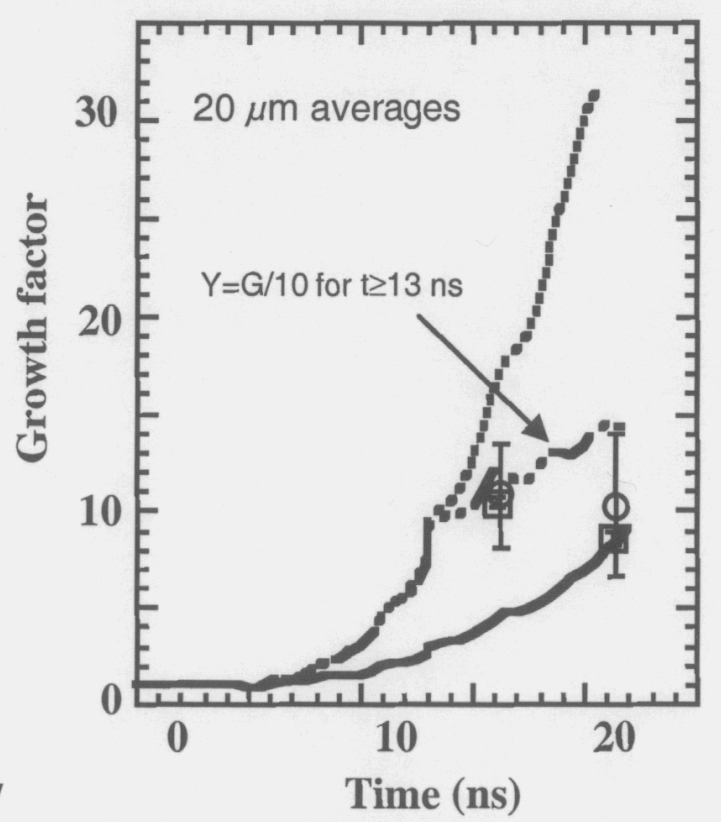

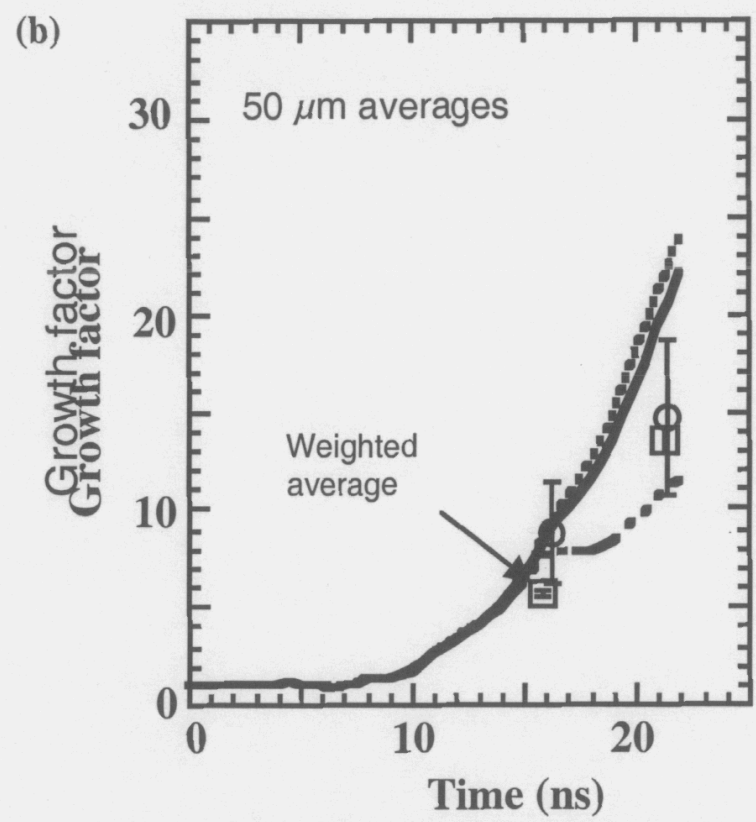

(d)

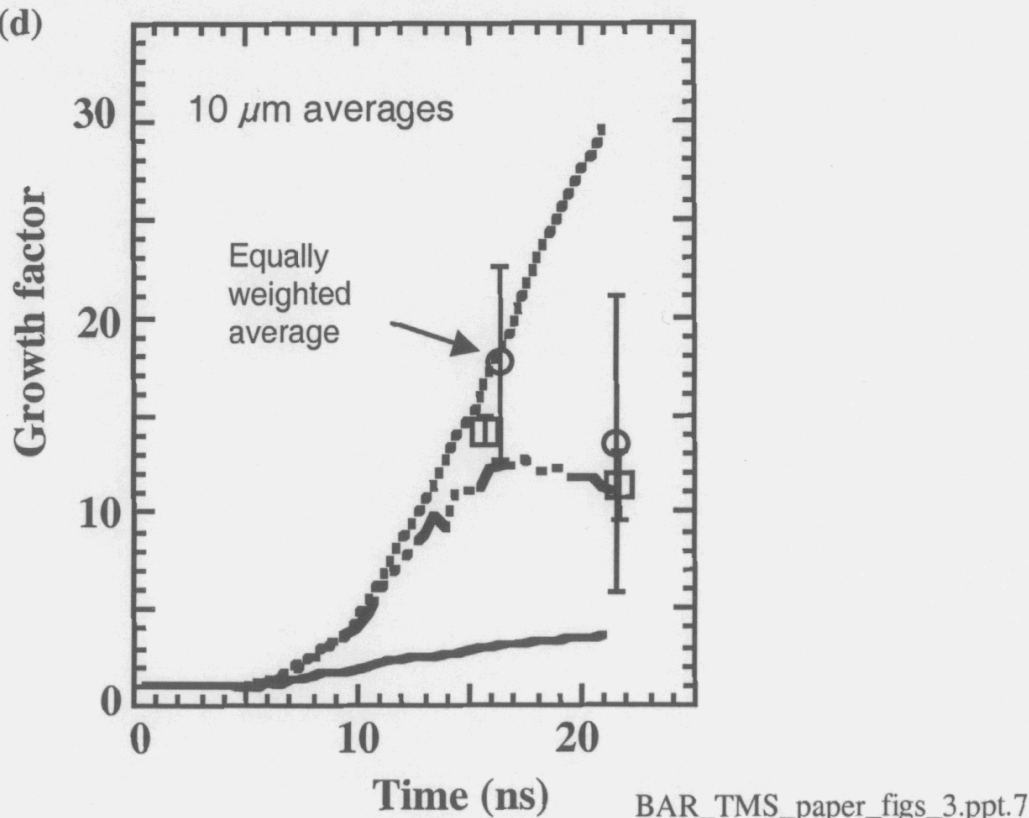




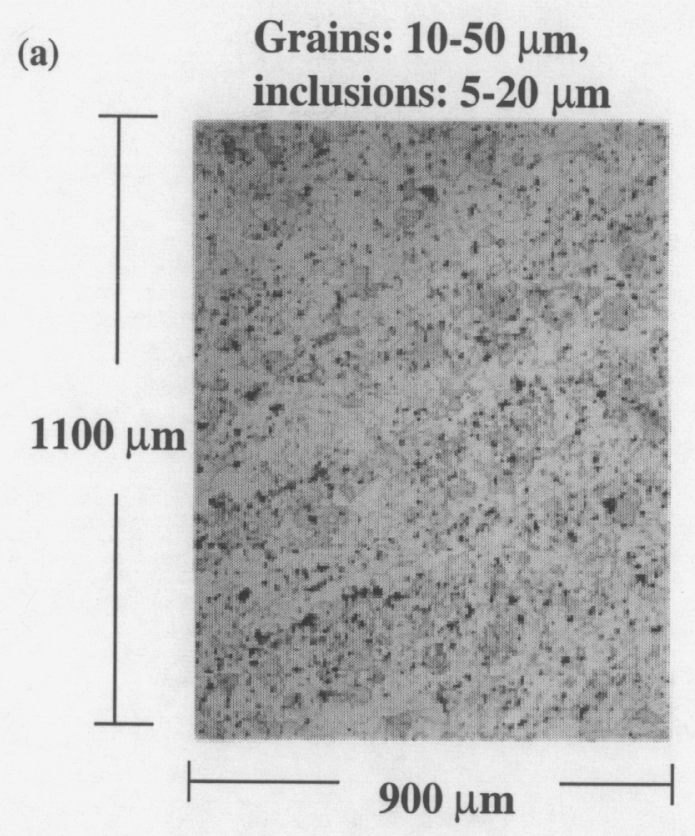

(b)

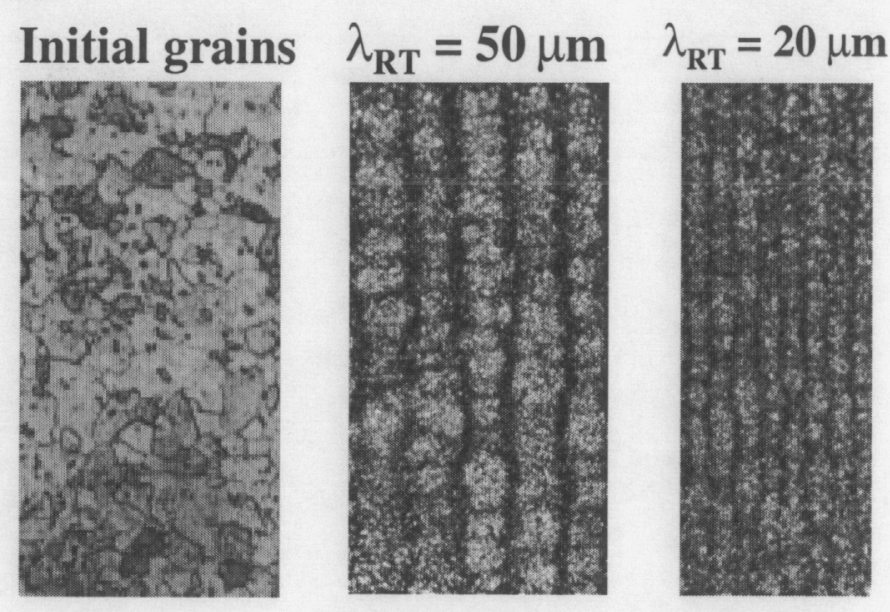

(d)

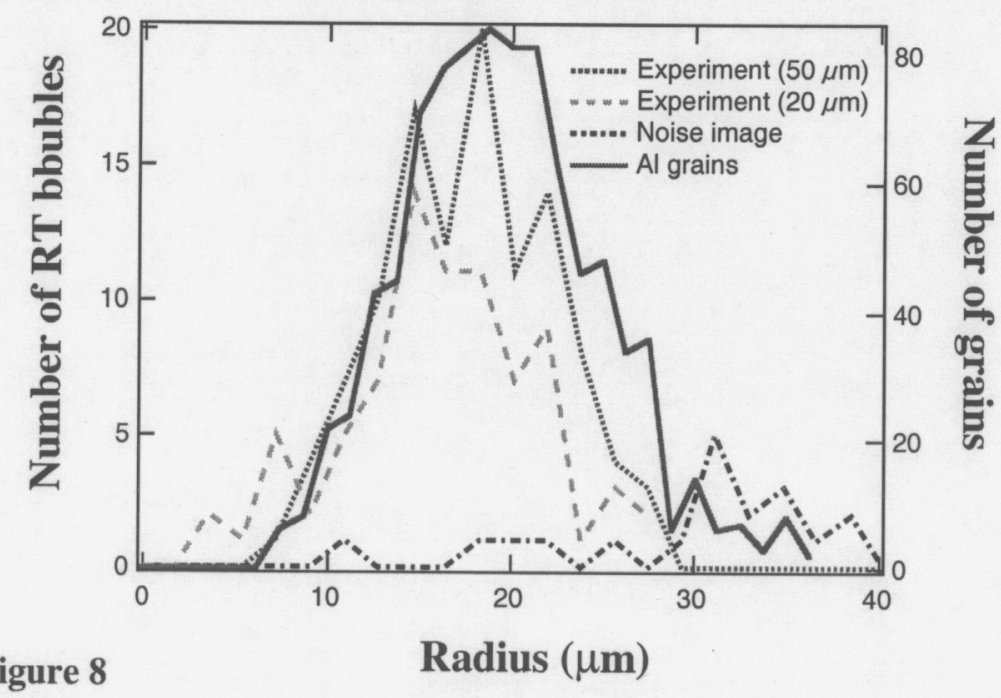

(c)

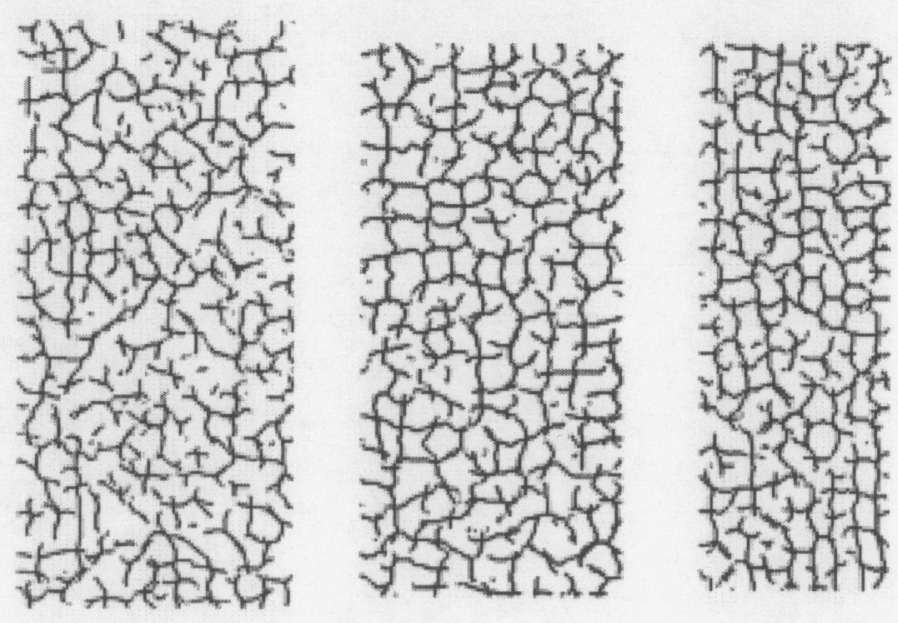



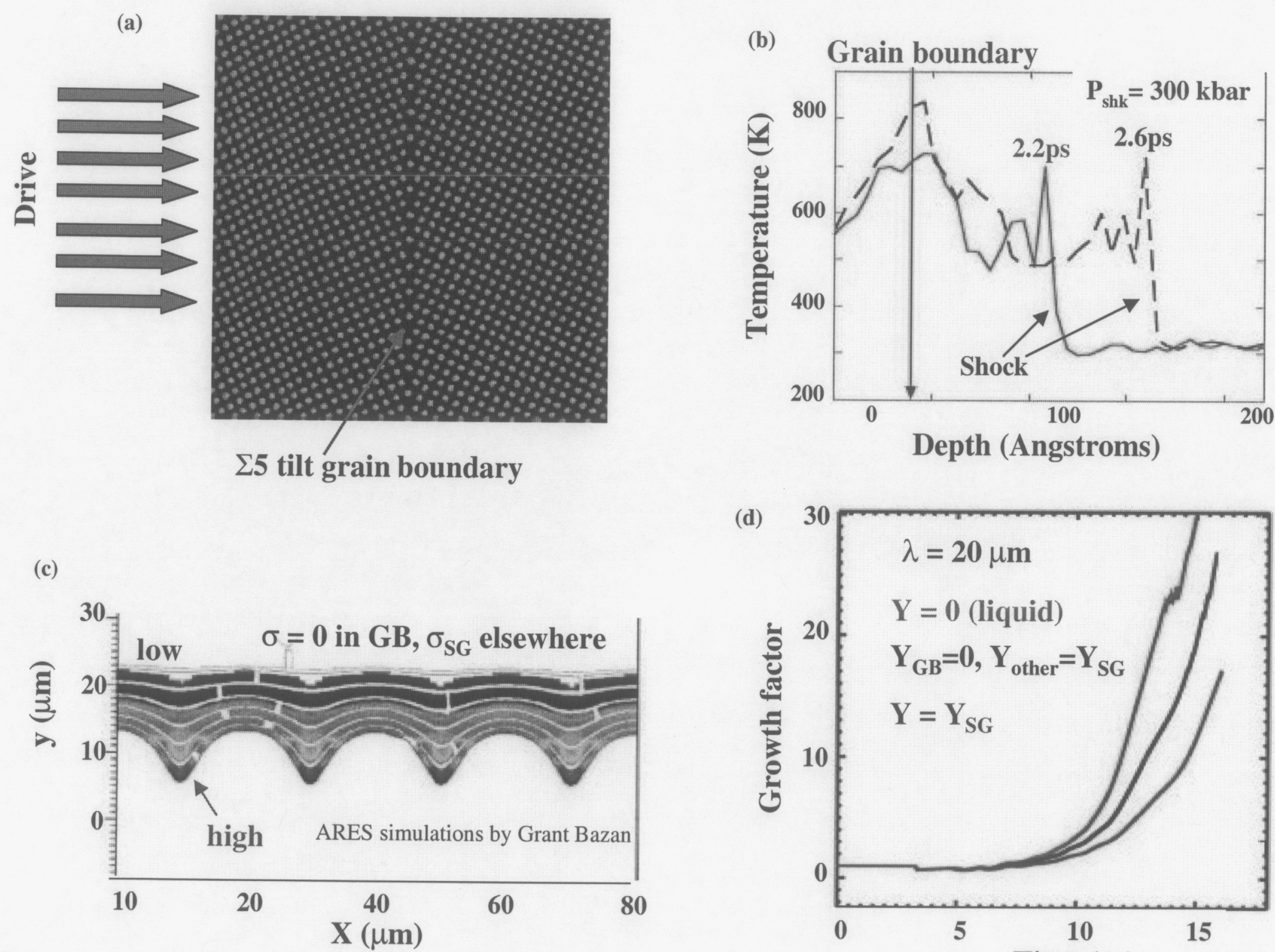

Figure 9

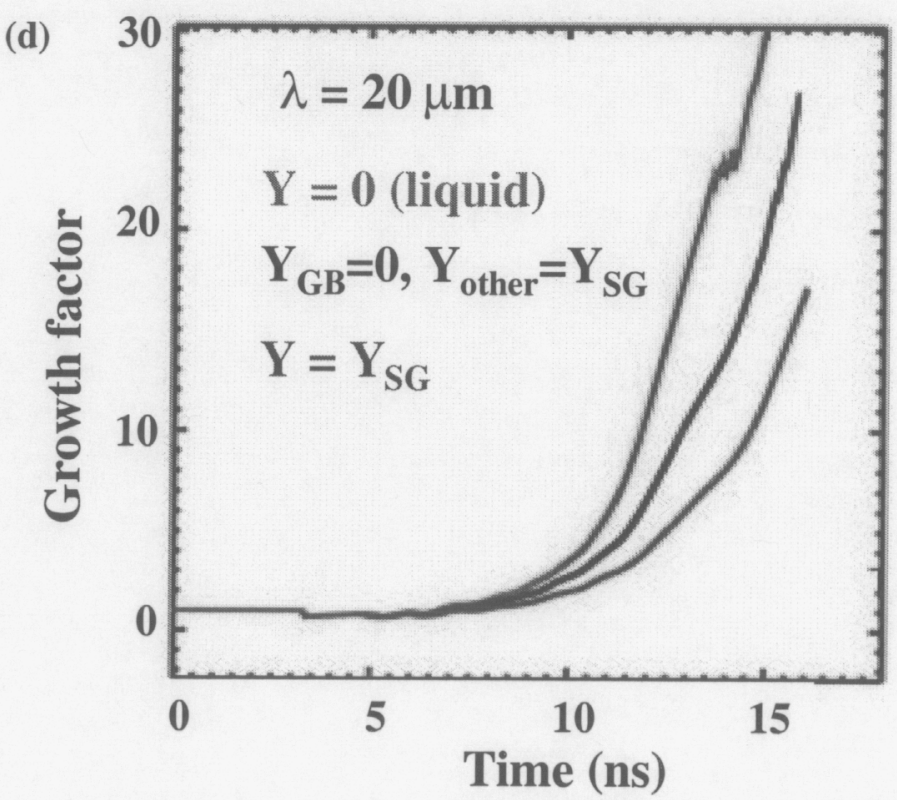

BAR_TMS_paper_figs_3.ppt.9 
(a)

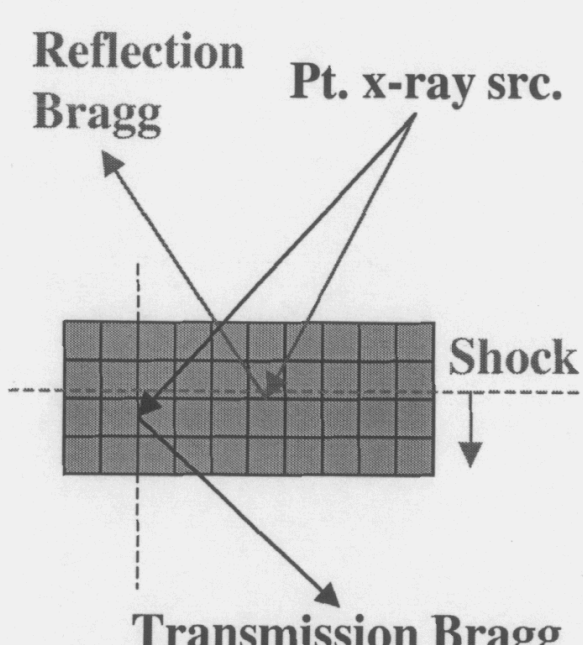

Transmission Bragg

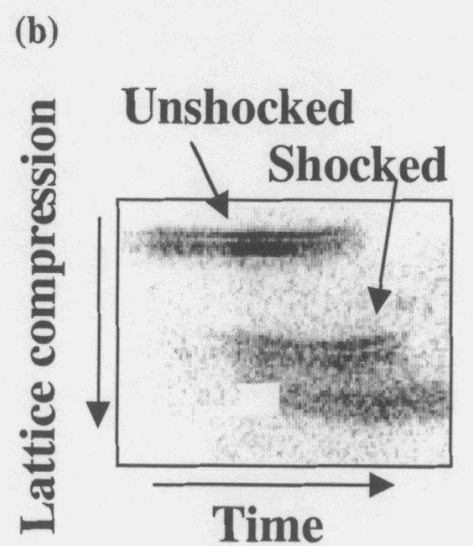

(c)

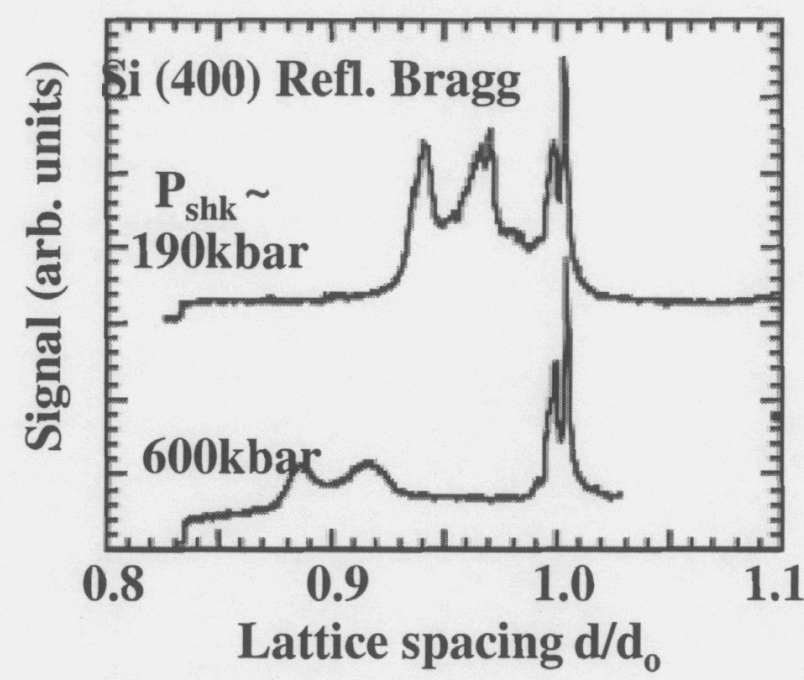

(d)

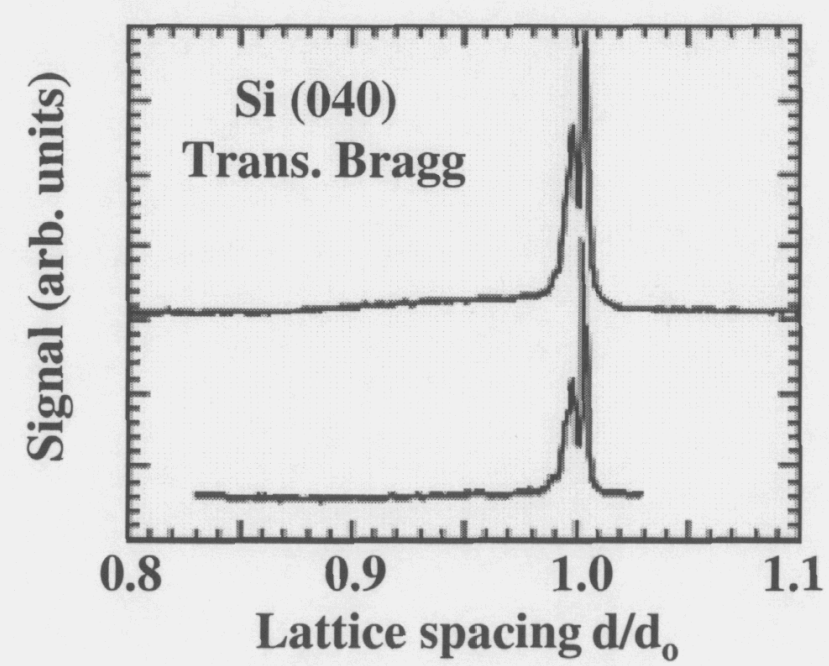

(e)

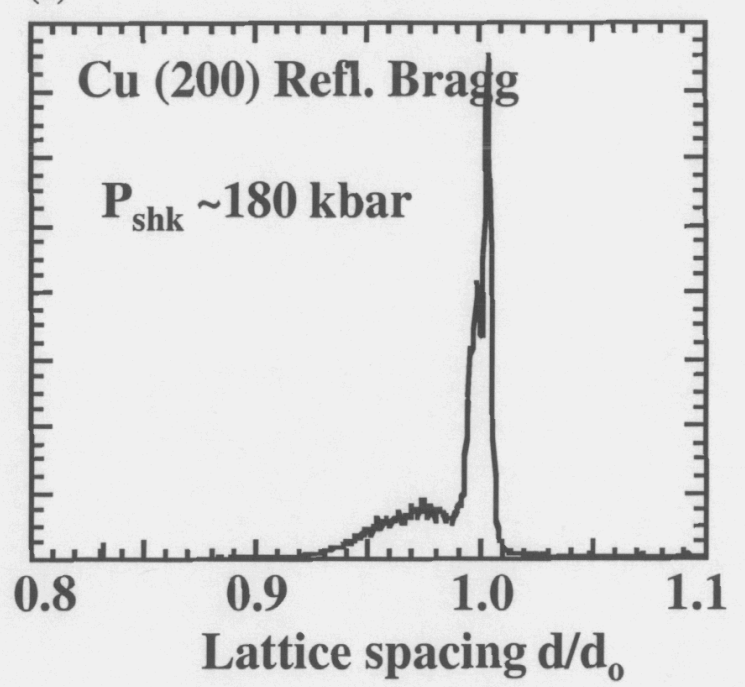

(f)

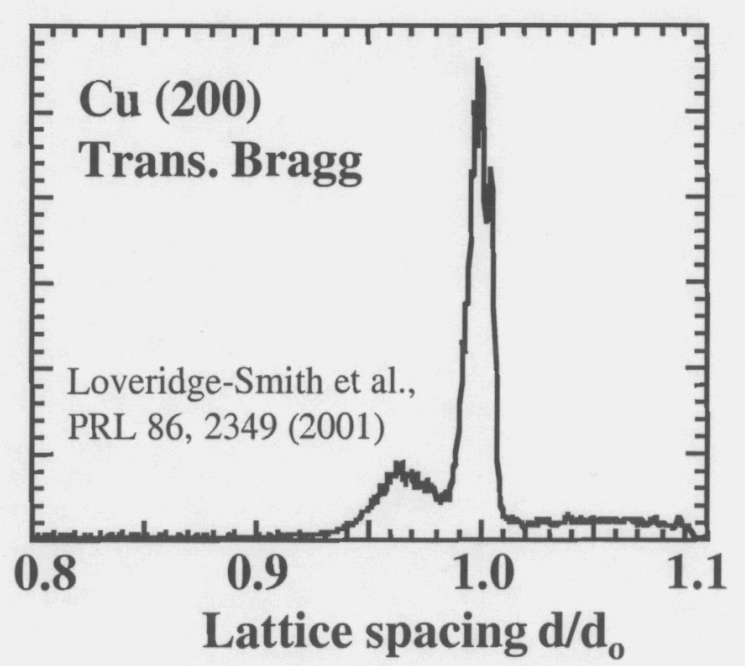

Figure 10 
(a)

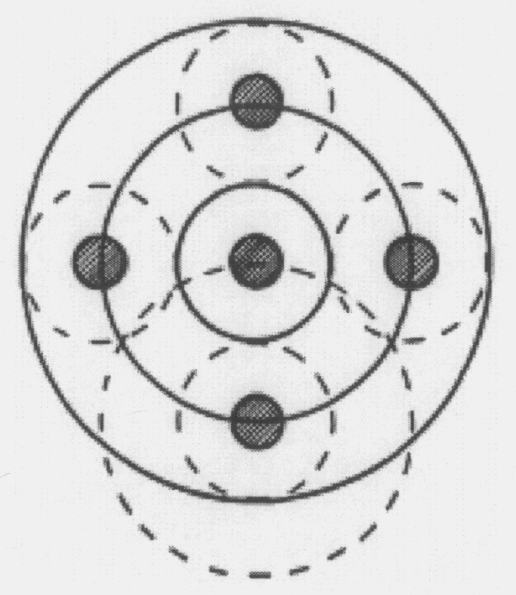

(c)

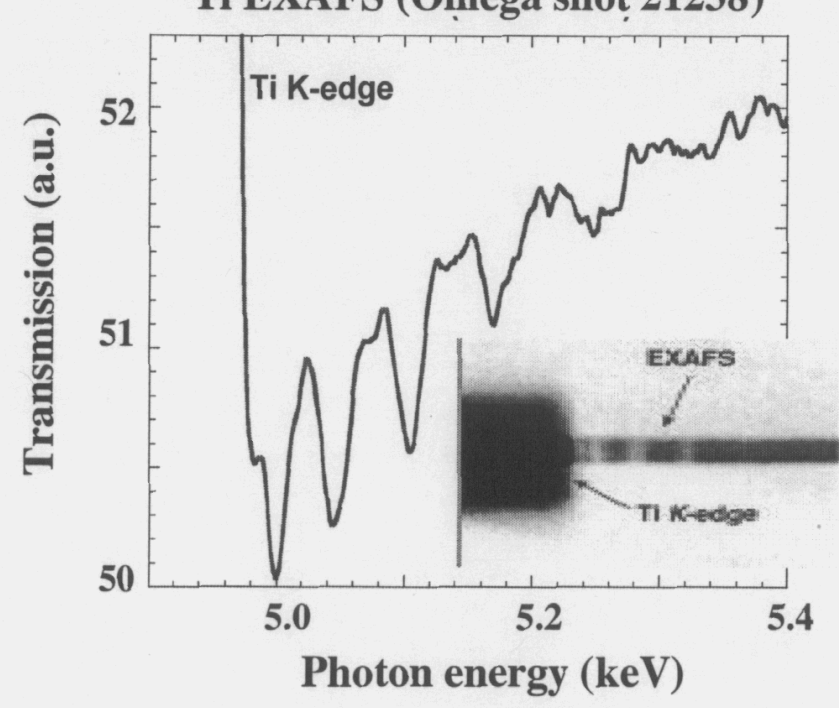

X-ray
(b) spectrometer (incident)

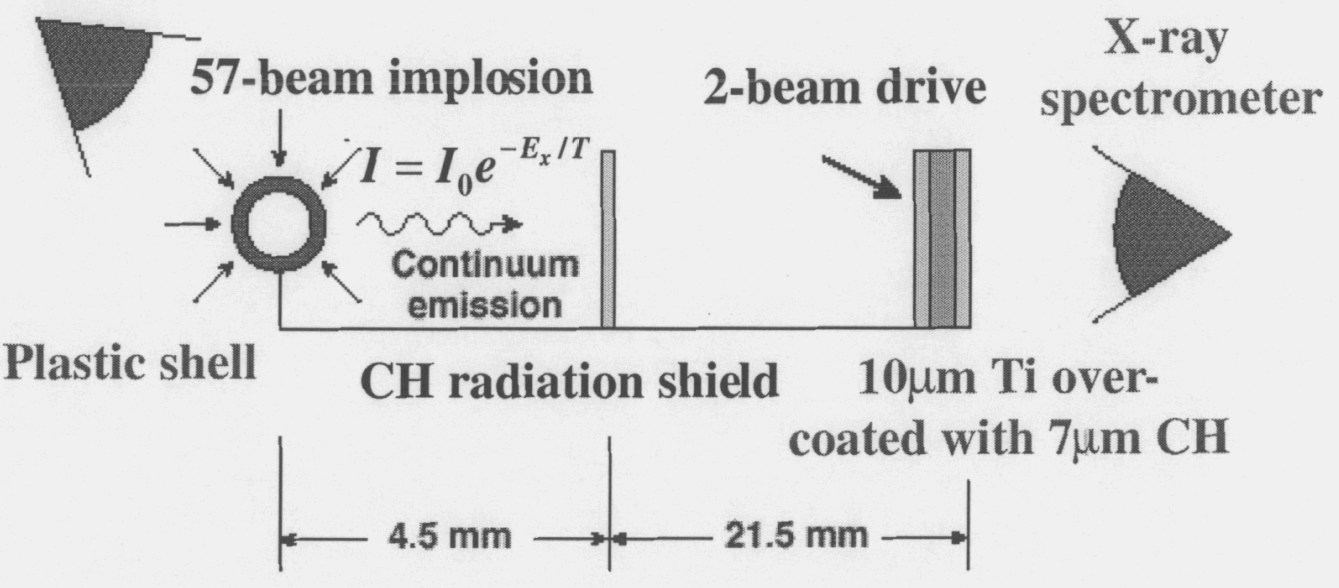

(d)

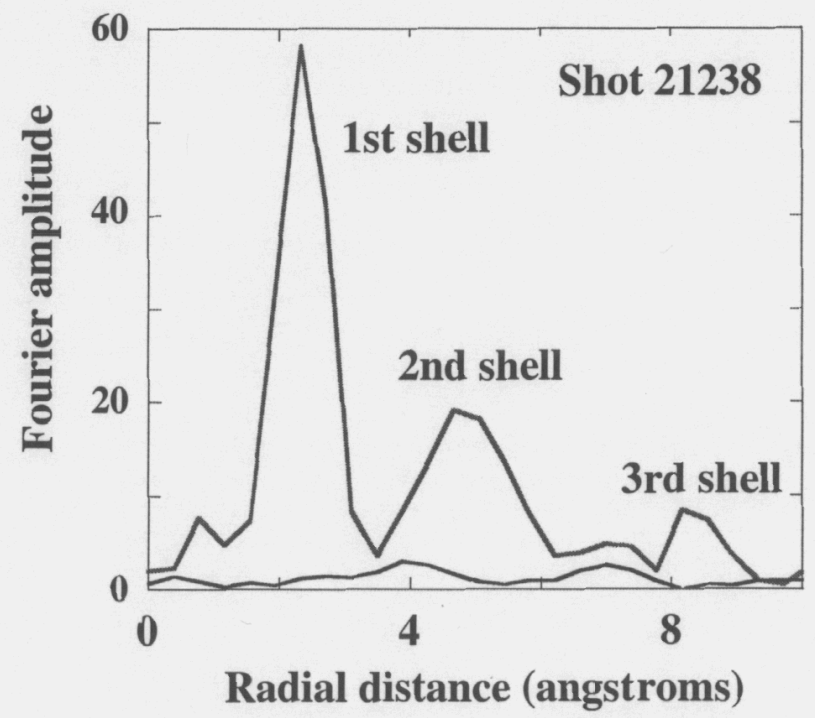

BAR_TMS_paper_figs_3.ppt.11 

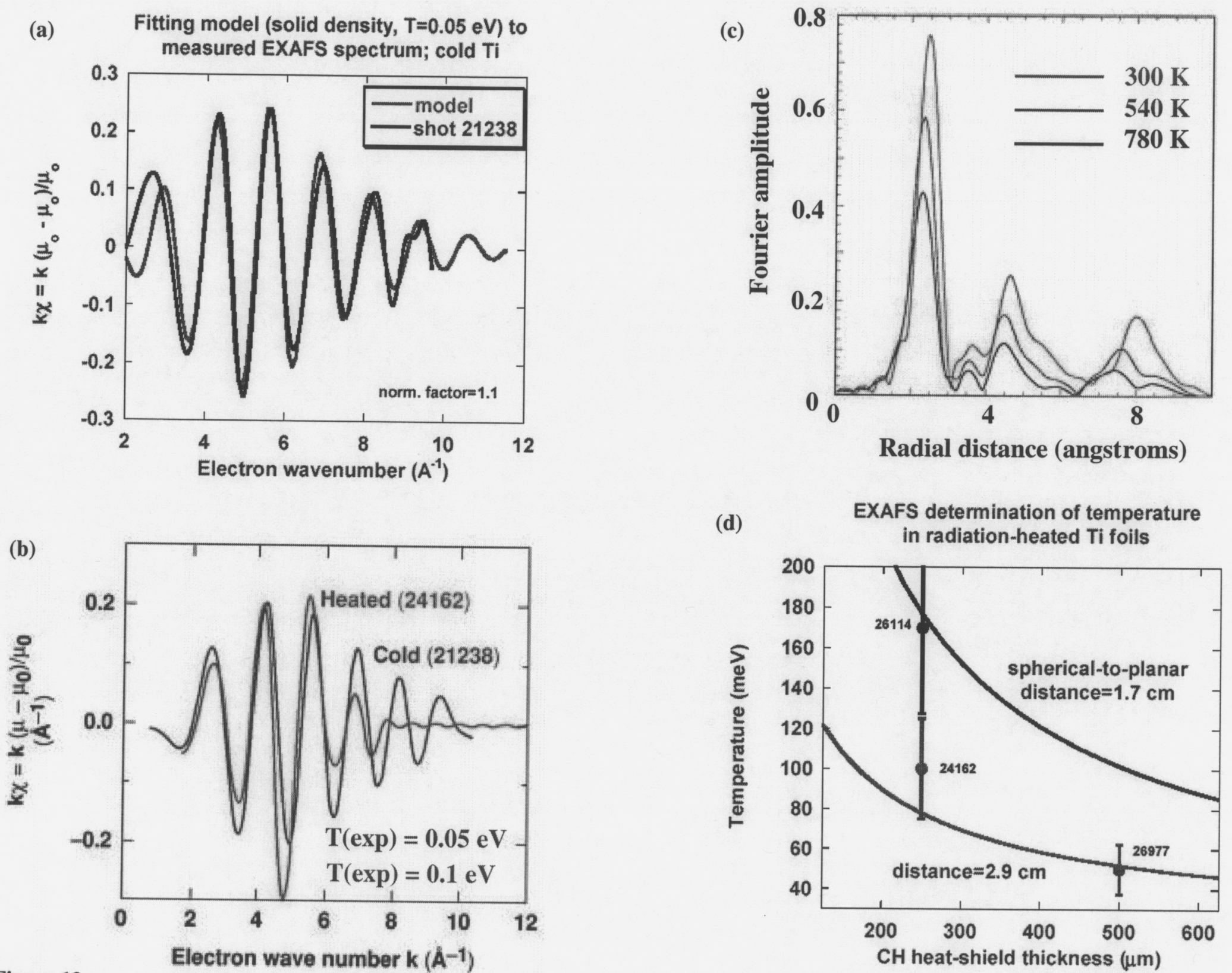

Figure 12 


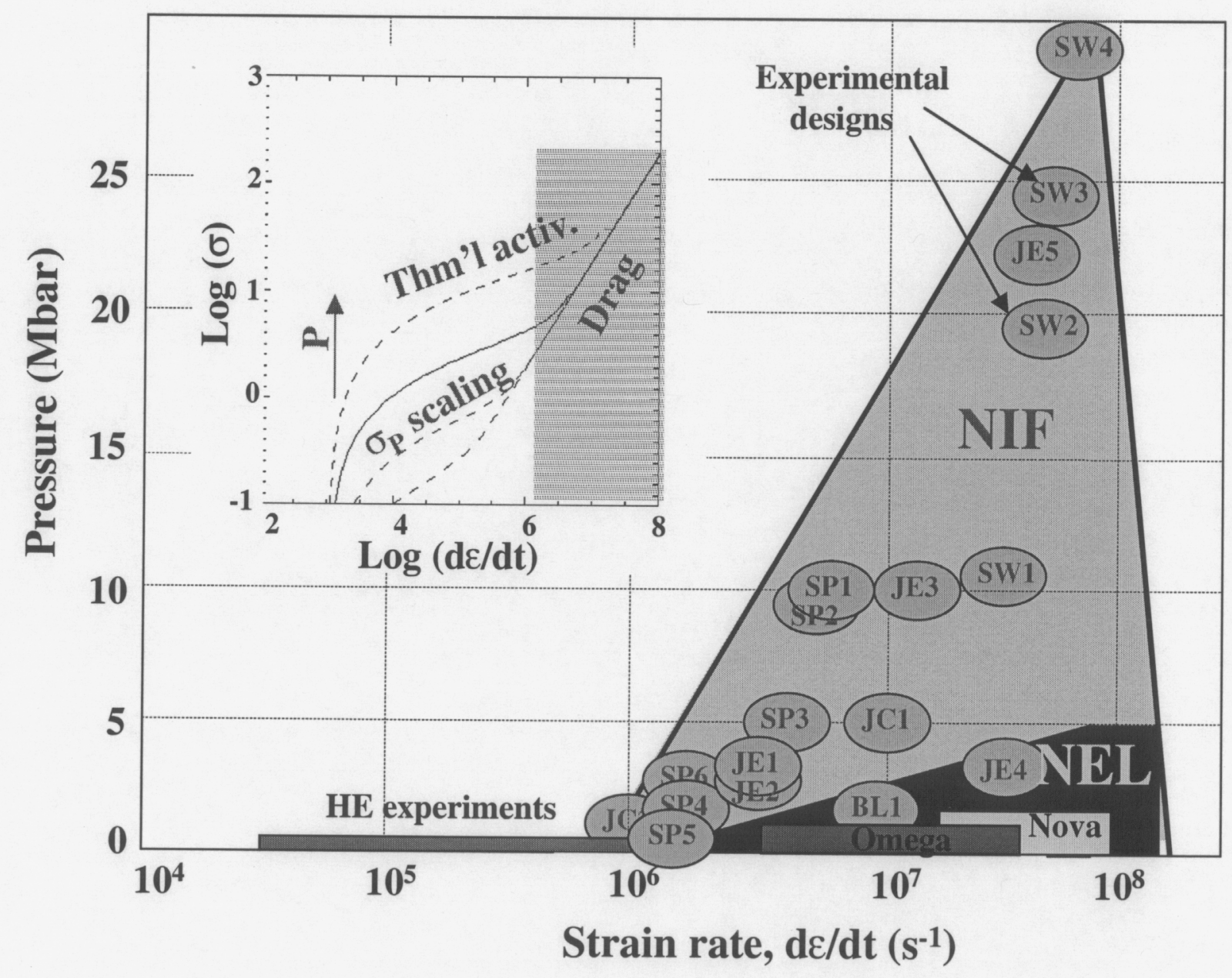

Figure 13 